\title{
Symmetry-Preserving Observers
}

\author{
Silvère Bonnabel, Philippe Martin, and Pierre Rouchon
}

\begin{abstract}
This paper presents the theory of invariant observers, i.e, symmetry-preserving observers. We consider an observer to consist of a copy of the system and a correction term, and we propose a constructive method (based on the Cartan moving-frame method) to find all the symmetry-preserving correction terms. The construction relies on an invariant frame (a classical notion) and on an invariant output-error, a less standard notion precisely defined here. Using the theory we build three non-linear observers for three examples of engineering interest: a non-holonomic car, a chemical reactor, and an inertial navigation system. For each example, the design is based on physical symmetries and the convergence analysis relies on the use of invariant state-errors, a symmetry-preserving way to define the estimation error.
\end{abstract}

Index Terms-Inertial navigation, moving frame, non-linear observer, symmetries.

\section{INTRODUCTION}

$\mathbf{S}$ YMMETRIES have been used in control theory for feedback design and optimal control, see for instance [10], [11], [15], [19]-[21] but much less for observer design [2], [3], [12]-[14]. In this paper we use symmetries for observer design and we develop a theory of invariant observers. The theoretical contribution of the paper is the following: for the smooth system with state $x$, input $u$ and output $y$, invariance under the action of a Lie group $G$ is defined and corresponds to a separate action of $G$ on the state-space, on the input-space and on the output space. Invariance means that the dynamics $(d / d t) x=f(x, u)$ and the output map $y=h(x, u)$ remain unchanged by a change of state, input, and output coordinates corresponding to the action of $G$. We define invariance for an asymptotic Luenberger non-linear observer under the action of $G$ similarly, where the group acts also on the estimated space and the estimated output in a similar way. When the group dimension does not exceed the state dimension we propose (theorem 1) a constructive design of the invariant observer. This construction is based on an invariant frame and an invariant output-error. Such invariant output-errors (definition 8) are introduced here for the first time and can be computed via Cartan's moving frame method (theorem 2). We show how to transform a locally convergent asymptotic observer around an equilibrium point into an invariant one with the same first order approximation. To deal with convergence issues, we introduce

Manuscript received February 09, 2007; revised October 15, 2007 and May 22, 2008. Current version published December 10, 2008. Recommended by Associate Editor M. Xiao.

S. Bonnabel is with the Montefiore Institute, University of Liège, Liège B-4000, Belgium (e-mail: bonnabel@montefiore.ulg.ac.be).

P. Martin and P. Rouchon are with the Centre Automatique et Systèmes, École des Mines de Paris, Paris 75272, France (e-mail: philippe. martin@ensmp.fr; pierre.rouchon@ensmp.fr).

Digital Object Identifier 10.1109/TAC.2008.2006929 invariant state-errors. We believe these state-errors play a key role in the convergence analysis.

The theory is motivated by three non-linear examples of engineering interest: a non-holonomic car, an exothermic chemical reactor and a velocity-aided inertial navigation system. In each case the symmetries have an obvious physical interpretation. For the first example we propose a non-linear observer which converges for any initial condition except one (theorem 4). For the second, we design a non-linear globally convergent observer (theorem 5). For the third, the observer is locally convergent around any system trajectory. Moreover the global behavior is independent of the system trajectory (theorem 6). This theory may be applied to many other systems such as those treated in [9], [12], [13] where the invariance relative to the choice of the reference 3D-frame is exploited in observer design and convergence analysis.

The content of this paper is as follows: in Section II, we define invariant systems and invariant pre-observers. The general form of an invariant pre-observer is given in theorem 1: it relies on invariant output errors and invariant vector fields. Their explicit construction relies on the moving frame method [17], which is summarized in Section II-B. Around an equilibrium, we prove it is always possible to build an invariant observer whose linear tangent approximation is any linear asymptotic observer of the Luenberger type. To study the convergence, we introduce the notion of invariant state error, a state error based on symmetries. Indeed the usual error $\hat{x}-x$ does not preserve the symmetries in general. This error obeys a differential system where only the invariant part of the system trajectory appears (theorem 3). This property reduces the dimension of the convergence problem and appears to play a crucial role in the examples. In Section III, we study in detail three physical examples.

A summary of the state of the art results on symmetries of dynamic systems can be found in the monograph [18]. The notion of invariant observer and invariant output error can be found in [1], [2]. Other preliminary results presented in this paper can be found in [6]-[8].

\section{INVARIANT SYSTEMS, OBSERVERS AND ERRORS}

\section{A. Invariant Systems and Equivariant Outputs}

Definition 1: Let $G$ be a Lie Group with identity $e$ and $\Sigma$ an open set (or more generally a manifold). A transformation group $\left(\phi_{g}\right)_{g \in G}$ on $\Sigma$ is a smooth map

$$
(g, \xi) \in G \times \Sigma \mapsto \phi_{g}(\xi) \in \Sigma
$$

such that:

- $\phi_{e}(\xi)=\xi$ for all $\xi$

- $\phi_{g_{2}}\left(\phi_{g_{1}}(\xi)\right)=\phi_{g_{2} g_{1}}(\xi)$ for all $g_{1}, g_{2}, \xi$.

Note $\phi_{g}$ is by construction a diffeomorphism on $\Sigma$ for all $g$. The transformation group is local if $\phi_{g}(\xi)$ is defined only when 
$g$ lies sufficiently near $e$. In this case the transformation law $\phi_{g_{2}}\left(\phi_{g_{1}}(\xi)\right)=\phi_{g_{2} g_{1}}(\xi)$ is valid only when it makes sense. In this section, all the results are local, since they are based on constant rank assumptions. We thus consider only local transformation groups acting on open sets. When we say "for all $g$ " we thus mean "for all $g$ sufficiently near the identity $e$ of $G$ "; in the same way "for all $\xi$ " usually means "for all generic $\xi$ in $\Sigma$ ". We systematically use these stylistic shortcuts to improve readability.

Consider now the smooth system

$$
\begin{aligned}
\frac{d}{d t} x & =f(x, u) \\
y & =h(x, u)
\end{aligned}
$$

where $x$ belongs to an open subset $\mathcal{X} \subset \mathbb{R}^{n}, u$ to an open subset $\mathcal{U} \subset \mathbb{R}^{m}$ and $y$ to an open subset $\mathcal{Y} \subset \mathbb{R}^{p}, p \leq n$.

We assume the signals $u(t), y(t)$ known. $y$ is the measured output. $u$ is measured or known. It can be a control input, a measured perturbation and it can also incorporate the model parameters. by

Consider the local group of transformations on $\mathcal{X} \times \mathcal{U}$ defined

$$
(X, U)=\left(\varphi_{g}(x), \psi_{g}(u)\right)
$$

where $\varphi_{g}$ and $\psi_{g}$ are local diffeomorphisms. Note that $\varphi_{g}$ acts on $\mathcal{X}$ and $\psi_{g}$ acts on $\mathcal{U}$. If $u$ is the time $(u(t)=t), \psi_{g}$ is the identity function. The two following definitions are inspired from [15].

Definition 2: The system $(d / d t) x=f(x, u)$ is $G$-invariant if $f\left(\varphi_{g}(x), \psi_{g}(u)\right)=D \varphi_{g}(x) \cdot f(x, u)$ for all $g, x, u$.

The property also reads $(d / d t) X=f(X, U)$, i.e., the system remains unchanged under the transformation (3).

Definition 3: The output $y=h(x, u)$ is $G$-equivariant if there exists a transformation group $\left(\varrho_{g}\right)_{g \in G}$ on $\mathcal{Y}$ such that $h\left(\varphi_{g}(x), \psi_{g}(u)\right)=\varrho_{g}(h(x, u))$ for all $g, x, u$.

With $(X, U)=\left(\varphi_{g}(x), \psi_{g}(u)\right)$ and $Y=\varrho_{g}(y)$, the definition means $Y=h(X, U)$. The two previous definitions can be illustrated by the commutative diagram

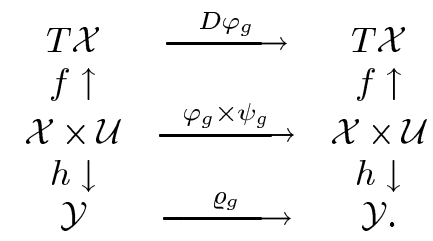

\section{B. The Moving Frame Method, Invariant Vector Fields, Base and Fiber Coordinates}

1) Moving Frame Method: This paragraph is independent of the rest of the paper. It is a recap of the general presentation of [17, theorem 8.25]). Take a r-dimensional transformation group $G$ acting on $\Sigma \subset \mathbb{R}^{s}$ via the diffeomorphisms $\left(\phi_{g}\right)_{g \in G}$ such that $r \leq s$. We suppose that $\partial_{g} \phi_{g}$ has full rank $r:=\operatorname{dim} G$ at the point $\left(e, \xi^{0}\right) \in G \times \Sigma$. We can then split $\phi_{g}$ into $\left(\phi_{g}^{a}, \phi_{g}^{b}\right)$ with respectively $r$ and $s-r$ components so that $\phi_{g}^{a}$ is invertible with respect to $g$ around $\left(e, \xi^{0}\right)$. The normalization equations are obtained setting

$$
\phi_{g}^{a}(\xi)=c
$$

with $c$ a constant in the range of $\phi^{a}$. The implicit function theorem ensures the existence of the local solution $g=\gamma(\xi)$ (the map $\gamma: \Sigma \rightarrow G$ is known as the moving frame). Thus

$$
\phi_{\gamma(\xi)}^{a}(\xi)=c .
$$

One can also say $\left\{\phi_{e}^{a}(\xi)=c\right\}$ defines a coordinate cross-section to the orbits, and $g=\gamma(\xi)$ is the unique group element that maps $\xi$ to the cross-section. Finally, we get a complete set $J$ of $s-r$ functionally independent invariants by substituting $g=$ $\gamma(\xi)$ into the remaining transformation rules

$$
J(\xi):=\phi_{\gamma(\xi)}^{b}(\xi) .
$$

The invariance property means $J\left(\phi_{g}(\xi)\right)=J(\xi)$ for all $g, \xi$. To prove it let $\zeta=\phi_{g}(\xi)$. We have $\phi_{\gamma\left(\phi_{g}(\xi)\right)}^{a}\left(\phi_{g}(\xi)\right)=\phi_{\gamma(\zeta)}^{a}(\zeta)=$ c. But the group composition implies $\phi_{\gamma\left(\phi_{g}(\xi)\right)}^{a}\left(\phi_{g}(\xi)\right)=$ $\phi_{\gamma\left(\phi_{g}(\xi)\right) g}^{a}(\xi)$. Thus $\phi_{\gamma\left(\phi_{g}(\xi)\right) g}^{a}(\xi)=c$ which proves by unicity of $\gamma(\xi) \in G$

$$
\gamma\left(\phi_{g}(\xi)\right) g=\gamma(\xi)
$$

which is the main property (equivariance) of the moving frame. We have indeed using (4)

$$
J\left(\phi_{g}(\xi)\right)=\phi_{\gamma\left(\phi_{g}(\xi)\right)}^{b}\left(\phi_{g}(\xi)\right)=\phi_{\gamma\left(\phi_{g}(\xi)\right) g}^{b}(\xi)=J(\xi)
$$

Moreover any other local invariant $J^{\prime}$, i.e, any real-valued function $J^{\prime}$ which verifies $J^{\prime}\left(\phi_{g}(\xi)\right)=J^{\prime}(\xi)$ for all $g, \xi$ can be written as a function of the complete set of invariants: $J^{\prime}=$ $\mathcal{H}(J)$.

2) Basic Assumptions: In the sequel we consider a $G$-invariant system $(d / d t) x=f(x, u)$ with a $G$-equivariant output $y=h(x, u)$. We let $r \leq n$ be the dimension of the group $G$. We systematically assume for each $x$, the mapping $g \mapsto \varphi_{g}(x)$ is full rank.

3) Invariant Vector Fields and Invariant Frame: The moving frame method allows us to build invariant frames.

Definition 4: A vector field $w$ on $\mathcal{X}$ is said to be G-invariant if the system $(d / d t) x=w(x)$ is invariant. This means $w\left(\varphi_{g}(x)\right)=D \varphi_{g}(x) \cdot w(x)$ for all $g, x$.

Definition 5: An invariant frame $\left(w_{1}, \ldots, w_{n}\right)$ on $\mathcal{X}$ is a set of $\mathrm{n}$ linearly point-wise independent G-invariant vector fields, i.e $\left(w_{1}(x), \ldots, w_{n}(x)\right)$ is a basis of the tangent space to $\mathcal{X}$ at $x$.

We are now going to explain how to build an invariant frame. We follow [16], theorem 2.84 and we now apply the moving frame method to the particular case: $\Sigma=\mathcal{X}$, and $\left(\phi_{g}\right)_{g \in G}=$ $\left(\varphi_{g}\right)_{g \in G}$ and the normalization equations $\varphi_{g}^{a}(x)=c$ give $g=$ $\gamma(x)$.

Lemma 1: The vector fields defined by

$$
w_{i}(x):=\left(D \varphi_{\gamma(x)}(x)\right)^{-1} \cdot \frac{\partial}{\partial x_{i}}, \quad i=1, \ldots, n
$$

where $\left(\left(\partial / \partial x_{1}\right), \ldots,\left(\partial / \partial x_{n}\right)\right)$ is the canonical frame of $\mathcal{X}$, form an invariant frame. ${ }^{1}$

Proof: They are clearly point-wise linearly independent. Each $w_{i}$ is invariant because for any group element $b$ we have

${ }^{1}$ One could take any basis $\left(e_{1}, \ldots, e_{n}\right)$ of $\mathcal{X}$ instead of the canonical frame. 
- $w_{i}\left(\varphi_{b}(x)\right)=\left(D \varphi_{\gamma\left(\varphi_{b}(x)\right)}\left(\varphi_{b}(x)\right)\right)^{-1} \cdot\left(\partial / \partial x_{i}\right)$ and thus

$$
\begin{aligned}
& \left(D \varphi_{b}(x)\right)^{-1} \cdot w_{i}\left(\varphi_{b}(x)\right) \\
& \quad=\left[D \varphi_{\gamma\left(\varphi_{b}(x)\right)}\left(\varphi_{b}(x)\right) D \varphi_{b}(x)\right]^{-1} \cdot \frac{\partial}{\partial x_{i}}
\end{aligned}
$$

- for $c, d \in G$ we have $\varphi_{c}\left(\varphi_{d}\right)(x)=\varphi_{c d}(x)$; thus

$$
D \varphi_{c}\left(\varphi_{d}(x)\right) D \varphi_{d}(x)=D \varphi_{c d}(x)
$$

Thus, with $c=\gamma\left(\varphi_{b}(x)\right)$ and $d=b$, we have

$$
D \varphi_{\gamma\left(\varphi_{b}(x)\right)}\left(\varphi_{b}(x)\right) D \varphi_{b}(x)=D \varphi_{\gamma\left(\varphi_{b}(x)\right) b}(x)
$$

- since $\gamma\left(\varphi_{b}(x)\right) b \equiv \gamma(x)((4))$, we have (corresponding to definition 4

$$
\left(D \varphi_{b}(x)\right)^{-1} \cdot w_{i}\left(\varphi_{b}(x)\right)=\left(D \varphi_{\gamma(x)}(x)\right)^{-1} \cdot \frac{\partial}{\partial x_{i}}=w_{i}(x)
$$

4) Base and Fiber Coordinates: We introduce base and fiber coordinates which are useful local coordinates to express G-invariant systems of definition 2 . Under the basic assumptions above, the moving frame method provides a set of fundamental local invariants $z_{b} \in \mathbb{R}^{n-r}$ of the group action on $\mathcal{X}$ alone. Complete it with $z_{a} \in \mathbb{R}^{r}$ so that $\left(z_{a}, z_{b}\right)$ form coordinates of $\mathcal{X}$. These coordinates are called fiber $\left(z_{a}\right)$ and base $\left(z_{b}\right)$ coordinates (see [17]). One can always choose $z_{a}$ such that for any $g \in G$ the group transformation reads $\varphi_{g}\left(z_{a}, z_{b}\right)=\left(\varpi\left(z_{a}\right), z_{b}\right)$ with $g \mapsto \varpi_{g}(x)$ invertible for all $x \in \mathbb{R}^{r}$. Let $\gamma$ be the moving frame which maps $z=\left(z_{a}, z_{b}\right)$ to the coordinate cross-section $\left\{z_{a}=c\right\}$. The invariant dynamics of definition 2 writes locally in the new coordinates

$$
\begin{aligned}
\frac{d}{d t} z_{a} & =D \varpi_{\gamma(z)^{-1}} f_{a}\left(c, z_{b}, \psi_{\gamma(z)}(u)\right) \\
\frac{d}{d t} z_{b} & =f_{b}\left(c, z_{b}, \psi_{\gamma(z)}(u)\right)
\end{aligned}
$$

since the system is invariant. Example Section III-B illustrates the interest of such coordinates.

\section{Characterization of Invariant Pre-Observers}

Definition 6 (Pre-Observer): The system $(d / d t) \hat{x}=$ $F(\hat{x}, u, y)$ is a pre-observer of (1), (2) if for all $x, u$ $F(x, u, h(x, u))=f(x, u)$.

The definition does not deal with convergence; if moreover $\hat{x}(t) \rightarrow x(t)$ as $t \rightarrow+\infty$ for every (close) initial conditions, the pre-observer is an (asymptotic) observer.

Definition 7: The pre-observer $(d / d t) \hat{x}=F(\hat{x}, u, y)$ is $G$ invariant if for all $g, \hat{x}, u, y$

$$
F\left(\varphi_{g}(\hat{x}), \psi_{g}(u), \varrho_{g}(y)\right)=D \varphi_{g}(\hat{x}) \cdot F(\hat{x}, u, y)
$$

The property also reads $(d / d t) \hat{X}=F(\hat{X}, U, Y)$, with $X=$ $\varphi_{g}(x), U=\psi_{g}(u)$ and $Y=\varrho_{g}(y)$. This means the pre-observer remains unchanged under the action of $G$ on each of the three spaces $\mathcal{X}, \mathcal{U}$, and $\mathcal{Y}$ via (resp.) $\varphi_{g}, \psi_{g}$ and $\varrho_{g}$. An invariant observer is an asymptotic G-invariant pre-observer.

The assumption that the output is G-equivariant is motivated by the following necessary condition (see [8]): if the pre-observer $(d / d t) \hat{x}=F(\hat{x}, u, y)$ is invariant and if the rank of $F$ versus $y$ is equal to $\operatorname{dim}(y)$, then, the output map $y$ is $G$-equivariant in the sense of definition 3 .

In general the "usual" output error $\hat{y}-y=h(\hat{x}, u)-y$ does not preserve the system geometry, hence it will not yield an invariant pre-observer. The key idea in order to build an invariant (pre-) observer is to use, as noticed in [2], an invariant output error instead of the usual output error.

Definition 8: The smooth map $(\hat{x}, u, y) \mapsto E(\hat{x}, u, y) \in \mathbb{R}^{p}$ is an invariant output error if

- the map $y \mapsto E(\hat{x}, u, y)$ is invertible for all $\hat{x}, u$;

- $E(\hat{x}, u, h(\hat{x}, u))=0$ for all $\hat{x}, u$;

- $E\left(\varphi_{g}(\hat{x}), \psi_{g}(u), \varrho_{g}(y)\right)=E(\hat{x}, u, y)$ for all $\hat{x}, u, y$.

The first and second properties mean $E$ is an "output error," i.e., it is zero if and only if $h(\hat{x}, u)=y$; the third property, which also reads $E(\hat{X}, U, Y)=E(\hat{x}, u, y)$, expresses invariance.

Theorem 1: $(d / d t) \hat{x}=F(\hat{x}, u, y)$ is a G-invariant pre-observer for the $G$-invariant system $(d / d t) x=f(x, u)$ with $G$-equivariant output $y=h(x, u)$ if and only if

$$
F(\hat{x}, u, y)=f(\hat{x}, u)+\sum_{i=1}^{n} \mathcal{L}_{i}(I(\hat{x}, u), E(\hat{x}, u, y)) w_{i}(\hat{x})
$$

where $E$ is an invariant output error, $(\hat{x}, u) \mapsto I(\hat{x}, u) \in$ $\mathbb{R}^{n+m-r}$ is a full-rank invariant function, the $\mathcal{L}_{i}$ 's are smooth functions such that for all $\hat{x}, \mathcal{L}_{i}(I(\hat{x}, u), 0)=0$, and $\left(w_{1}, \ldots, w_{n}\right)$ is an invariant frame.

Since each $\mathcal{L}_{i}$ is smooth and satisfies $\mathcal{L}_{i}(I, 0)=0$, we can write $\mathcal{L}_{i}(I, E)=\overline{\mathcal{L}}_{i}(I, E) E$ where $\overline{\mathcal{L}}_{i}(I, E)$ is a $1 \times p$ matrix with entries depending on $(I, E)$. Hence

$$
\begin{aligned}
\sum_{i=1}^{n} \mathcal{L}_{i}(I, E) w_{i} & =\sum_{i=1}^{n} w_{i}\left(\overline{\mathcal{L}}_{i}(I, E) E\right) \\
& =\left(\begin{array}{lll}
w_{1} & \cdots & w_{n}
\end{array}\right)\left(\begin{array}{c}
\overline{\mathcal{L}}_{1}(I, E) \\
\vdots \\
\overline{\mathcal{L}}_{n}(I, E)
\end{array}\right) E .
\end{aligned}
$$

The observer can thus be written in the form

$$
F(\hat{x}, u, y)=f(\hat{x}, u)+W(\hat{x}) \overline{\mathcal{L}}(I(\hat{x}, u), E(\hat{x}, u, y)) E(\hat{x}, u, y)
$$

where $W(\hat{x})=\left(w_{1}(\hat{x}), \ldots, w_{n}(\hat{x})\right)$ and $\overline{\mathcal{L}}$ is a $n \times p$ matrix whose entries depend on $(I, E)$. The observer can be thought of as a gain-scheduled observer with a $n \times p$ gain matrix $W \cdot \overline{\mathcal{L}}$ multiplied by the non-linear error $E$.

Notice the theorem says nothing about convergence but only deals with the structure of the pre-observer.

To prove theorem 1 we first prove the following theorem which ensures the existence of a (local) invariant output error. The proof is constructive and relies on the Cartan moving frame method (see Section II-B-1).

Theorem 2: We have the three following statements: 
- there is an invariant output error $(\hat{x}, u, y) \mapsto E(\hat{x}, u, y)$;

- there is a full-rank invariant function $(\hat{x}, u) \mapsto I(\hat{x}, u) \in$ $\mathbb{R}^{n+m-r}$ (a complete set of $\mathrm{n}+\mathrm{m}-\mathrm{r}$ independent scalar invariants);

- every other invariant output error reads

$$
\tilde{E}(\hat{x}, u, y)=\mathcal{L}(I(\hat{x}, u), E(\hat{x}, u, y)) .
$$

where $\mathcal{L}$ is any smooth function such that $\mathcal{L}(I, 0)=0$ and $E \mapsto \mathcal{L}(I, E)$ is invertible.

Proof: We apply the moving frame method (Section II-B-1) to another particular case: $\Sigma=\mathcal{X} \times \mathcal{U} \times \mathcal{Y}$, and $\phi_{g}$ is the composite transformation

$$
\phi_{g}(\hat{x}, u, y):=\left(\varphi_{g}(\hat{x}), \psi_{g}(u), \varrho_{g}(y)\right) .
$$

Since the action of $G$ on $\mathcal{X}$ is full rank we can split $\hat{x} \mapsto$ $\varphi_{g}(\hat{x})$ into $\varphi_{g}^{a}(\hat{x}) \in \mathbb{R}^{r}$, which is invertible with respect to $g$, and the remaining part $\varphi_{g}^{b}(\hat{x}) \in \mathbb{R}^{n-r}$. The $r$ normalization equations

$$
\varphi_{g}^{a}(\hat{x})=c
$$

can then be solved and give $g=\gamma(\hat{x})$, which can be substituted into the remaining equations to yield the complete set of $n+$ $m+p-r$ functionally independent invariants

$$
\begin{aligned}
I(\hat{x}, u) & :=\left(\varphi_{\gamma(\hat{x})}^{b}(\hat{x}), \psi_{\gamma(\hat{x})}(u)\right) \\
J_{h}(\hat{x}, y) & :=\varrho_{\gamma(\hat{x})}(y) .
\end{aligned}
$$

An invariant output error is then given by

$$
E(\hat{x}, u, y):=J_{h}(\hat{x}, h(\hat{x}, u))-J_{h}(\hat{x}, y) .
$$

Actually, since it is an invariant function of $\hat{x}, u$ and $y$, every invariant output error $\tilde{E}$ must have the form

$$
\begin{aligned}
\tilde{E}(\hat{x}, u, y) & =\mathcal{F}\left(I(\hat{x}, u), J_{h}(\hat{x}, y)\right) \\
& =\mathcal{F}\left(I(\hat{x}, u), J_{h}(\hat{x}, h(\hat{x}, u))-E(\hat{x}, u, y)\right) \\
& =\mathcal{L}(I(\hat{x}, u), E(\hat{x}, u, y)) .
\end{aligned}
$$

We used the fact that $J_{h}(\hat{x}, h(\hat{x}, u))$, which is by construction invariant, must be a function of $I(\hat{x}, u)$ (fundamental invariants of $\hat{x}$ and $u$ ).

We are now able to give the proof of theorem 1:

Proof: The vector field $F$ in the theorem clearly is a preobserver. Indeed with $y=h(x, u)$ we have

$$
\begin{aligned}
F(x, u, y) & =f(x, u)+\sum_{i=1}^{n} \mathcal{L}_{i}(I(x, u), E(x, u, y)) w_{i}(x) \\
& =f(x, u)+\sum_{i=1}^{n} \mathcal{L}_{i}(I(x, u), 0) w_{i}(x) \\
& =f(x, u)
\end{aligned}
$$

By construction, it is invariant.

Conversely, assume $(d / d t) \hat{x}=F(\hat{x}, u, y)$ is a G-invariant observer. It can be decomposed on the point-wise independent $w_{i}$ 's as

$$
F(\hat{x}, u, y)=\sum_{i=1}^{n} F_{i}(\hat{x}, u, y) w_{i}(\hat{x})
$$

where the $F_{i}$ 's are smooth functions. Since it is a pre-observer

$$
f(x, u)=F(x, u, h(x, u))=\sum_{i=1}^{n} F_{i}(x, u, h(x, u)) w_{i}(x) .
$$

Since it is a G-invariant pre-observer

$$
\begin{array}{r}
\sum_{i=1}^{n} F_{i}\left(\varphi_{g}(\hat{x}), \psi_{g}(u), \varrho_{g}(y)\right) w_{i}\left(\varphi_{g}(\hat{x})\right) \\
=D \varphi_{g}(\hat{x}) \cdot \sum_{i=1}^{n} F_{i}(\hat{x}, u, y) w_{i}(\hat{x})
\end{array}
$$

but the $w_{i}$ 's verify $D \varphi_{g}(\hat{x}) \cdot w_{i}(\hat{x})=w_{i}\left(\varphi_{g}(x)\right)$, hence

$$
F_{i}\left(\varphi_{g}(\hat{x}), \psi_{g}(u), \varrho_{g}(y)\right)=F_{i}(\hat{x}, u, y), \quad i=1, \ldots n .
$$

Therefore

$$
\begin{aligned}
& F(\hat{x}, u, y)=f(\hat{x}, u)+[F(\hat{x}, u, y)-f(\hat{x}, u)] \\
& \quad=f(\hat{x}, u)+\sum_{i=1}^{n}\left[F_{i}(\hat{x}, u, y)-F_{i}(\hat{x}, u, h(\hat{x}, u))\right] w_{i}(\hat{x})
\end{aligned}
$$

The functions $F_{i}(\hat{x}, u, y)-F_{i}(\hat{x}, u, h(\hat{x}, u))$ are clearly invariant; hence by theorem $2, F_{i}(\hat{x}, u, y)-F_{i}(\hat{x}, u, h(\hat{x}, u))=$ $\mathcal{L}_{i}(I(\hat{x}, u), E(\hat{x}, u, y))$.

\section{Invariant Pre-Observer: A Constructive Method}

Consider an invariant system (i.e, unchanged by transformation (3)) with equivariant output (definition 3).

a) Solve the normalization equations (8). Build an invariant error thanks to (11), and a complete set of scalar invariants $I$ thanks to (9).

b) Build an invariant frame thanks to (5).

c) The general form of all invariant pre-observers is given by theorem 1 . There is a convenient alternative form (7).

\section{E. Local Convergence Around an Equilibrium}

In this paragraph we prove it is always possible to turn an asymptotic observer with a local gain design into an invariant one with the same local behavior (see the chemical reactor of Section III-B). Indeed consider an equilibrium $(\bar{x}, \bar{u}, \bar{y})$ characterized by $f(\bar{x}, \bar{u})=0$ and $\bar{y}=h(\bar{x}, \bar{u})$. Assume that the linearized system around this equilibrium is observable. This means that the pair $(A, C)$ is observable where

$$
A=\frac{\partial f}{\partial x}(\bar{x}, \bar{u}), B=\frac{\partial f}{\partial u}(\bar{x}, \bar{u}), C=\frac{\partial h}{\partial x}(\bar{x}, \bar{u}), D=\frac{\partial h}{\partial u}(\bar{x}, \bar{u}) .
$$


Consider the following locally asymptotic observer:

$$
\frac{d}{d t} \hat{x}=f(\hat{x}, u)+L(\hat{y}-y)
$$

with $\hat{y}=h(\hat{x}, u)$, and where we have chosen the observer constant gain matrix $L$ such that $A+L C$ is a stable matrix. In general, such an observer is not invariant. One can build an invariant observer with the same linear-tangent approximation, i.e., a locally asymptotic observer of the form (7)

$$
\frac{d}{d t} \hat{x}=f(\hat{x}, u)+W(\hat{x}) \overline{\mathcal{L}}(I(\hat{x}, u), E(\hat{x}, u, y)) E(\hat{x}, u, y)
$$

with

$$
\begin{aligned}
& \frac{\partial F}{\partial \hat{x}}(\bar{x}, \bar{u}, \bar{y})=A+L C, \quad \frac{\partial F}{\partial u}(\bar{x}, \bar{u}, \bar{y})=B+L D, \\
& \frac{\partial F}{\partial y}(\bar{x}, \bar{u}, \bar{y})=-L .
\end{aligned}
$$

Let us suggest a possible choice for $\overline{\mathcal{L}}$ in order to satisfy the above conditions on $\partial F / \partial \hat{x}, \partial F / \partial x$ and $\partial F / \partial y$ at the equilibrium. Since $E(\hat{x}, u, \hat{y}) \equiv 0$, by differentiation versus $\hat{x}, u$ and $y$, we have at the equilibrium

$$
\frac{\partial E}{\partial \hat{x}}=-\frac{\partial E}{\partial y} C, \quad \frac{\partial E}{\partial u}=-\frac{\partial E}{\partial y} D .
$$

Let $V$ denote the $p \times p$ square invertible matrix $V=$ $\partial E / \partial y(\bar{x}, \bar{u}, \bar{y})$. Take for instance the constant matrix

$$
\overline{\mathcal{L}}=-W(\bar{x})^{-1} L V^{-1} .
$$

The choice proposed for $\overline{\mathcal{L}}$ is such that the above conditions (13) are fulfilled. We made an invariant observer with the same local behavior as (12).

\section{F. Invariant State-Error and Convergence Issue}

We have no general constructive procedure to design the gain functions $\mathcal{L}_{i}$ 's of theorem 1 in order to achieve systematic asymptotic convergence of $\hat{x}$ towards $x$ for any non-linear system possessing symmetries. Nevertheless the way the state estimation error is defined plays a key role in convergence analysis. Instead of the linear state-error $\hat{x}-x$, we will rather consider the following invariant state-error:

$$
\eta(x, \hat{x})=\varphi_{\gamma(x)}(\hat{x})-\varphi_{\gamma(x)}(x)
$$

where $\gamma(x)$ is defined as the solution of (8) with respect to $g$. Note that it is equivalent to choose $\hat{x}$ to make the normalization and consider $\eta(\hat{x}, x)=\varphi_{\gamma(\hat{x})}(x)-\varphi_{\gamma(\hat{x})}(\hat{x})$. Let $I(x, u)$ be a complete set of scalar invariants for the action of $G$ on $\mathcal{X} \times \mathcal{U}$. A remarkable result is that the (non-linear) error equation does not depend on the trajectory via the $(n+m)$-dimensional variable $(x, u)$ as usual, but via $I(x, u)$, i.e, $(n+m)-r$ scalar invariants:

Theorem 3: The dynamics of the invariant state-error $\eta(x, \hat{x})=\varphi_{\gamma(x)}(\hat{x})-\varphi_{\gamma(x)}(x)$ depends only on $\eta$ and scalar invariants depending on $x$ and $u$

$$
\frac{d}{d t} \eta=\Upsilon(\eta, I(x, u))
$$

for some smooth function $\Upsilon$ and where $I(x, u)$ is defined in theorem 2.

Proof: The error $\eta$ is an invariant: for all $g \in G$ we have $\eta\left(\varphi_{g}(x), \varphi_{g}(\hat{x})\right)=\eta(x, \hat{x})$. Thus $(d / d t) \eta\left(\varphi_{g}(x), \varphi_{g}(\hat{x})\right)=$ $(d / d t) \eta(x, \hat{x})$, i.e.

$$
\begin{aligned}
\partial_{1} \eta D \varphi_{g}(x) f(x, u) & +\partial_{2} \eta D \varphi_{g}(\hat{x}) F(\hat{x}, u, h(x, u)) \\
= & \partial_{1} \eta f(x, u)+\partial_{2} \eta F(\hat{x}, u, h(x, u))
\end{aligned}
$$

where $\partial_{i}$ denotes the partial differential relative to the $i$-th variable. Let $\sigma(\hat{x}, x, u)=(d / d t) \eta(x, \hat{x})=\partial_{1} \eta f(x, u)+$ $\partial_{2} \eta F(\hat{x}, u, h(x, u))$. The equality (14) expresses that $\sigma\left(\varphi_{g}(\hat{x}), \varphi_{g}(x), \psi_{g}(u)\right)=\sigma(\hat{x}, x, u)$. Since $\hat{x}=\varphi_{\gamma(x)^{-1}}(\eta+$ $\left.\varphi_{\gamma(x)}(x)\right), \sigma$ is an invariant function of the variables $(\eta, x, u)$. Since $\eta$ is an invariant, every invariant function of $(\eta, x, u)$ (in particular $(d / d t) \eta)$ is a function of $\eta$, and of a fundamental set of scalar invariants of $x$ and $u: I(x, u)$.

Such invariant coordinates are not unique. Any invariant function of $x, \hat{x}$ and $u$ equal to zero when $\hat{x}=x$ can be used as an invariant state-error to analyze convergence. Since it must be a function of the complete set of $2 n+m-r$ invariants $\left(I(x, u), \varphi_{\gamma(x)}(\hat{x})\right)$, it must be a function of $I(x, u)$ and of the invariant state-error $\eta(x, \hat{x}): \mathcal{F}(I(x, u), \eta(x, \hat{x}))$ where $\mathcal{F}(I, 0)=0$ for all $I$. All examples illustrate the interest of such special coordinates to analyze convergence.

\section{EXAMPLES}

\section{A. The Non-Holonomic Car}

Consider a non-holonomic car whose dynamics is the following:

$$
\begin{aligned}
\frac{d}{d t} x & =u \cos \theta, \quad \frac{d}{d t} y=u \sin \theta, \quad \frac{d}{d t} \theta=u v, \\
h(x, y, \theta) & =(x, y)
\end{aligned}
$$

where $u$ is the velocity and $v$ is a function of the steering angle. We suppose the output is the measurement of the position $h(x, y, \theta)=(x, y)$ (using a GPS for instance).

The system is independent of the origin and of the orientation of the frame chosen, i.e., it is invariant under the action of $G=S E(2)$, the group of rotations and translations. We make the identification $G=\mathbb{R}^{2} \times S^{1}$ thus any element of $G$ writes $\left(x_{g}, y_{g}, \theta_{g}\right) \in \mathbb{R}^{2} \times S^{1}$ and the group multiplication is

$$
\left(\begin{array}{l}
x_{g} \\
y_{g} \\
\theta_{g}
\end{array}\right) \cdot\left(\begin{array}{l}
x \\
y \\
\theta
\end{array}\right)=\left(\begin{array}{c}
x \cos \theta_{g}-y \sin \theta_{g}+x_{g} \\
x \sin \theta_{g}+y \cos \theta_{g}+y_{g} \\
\theta+\theta_{g}
\end{array}\right) .
$$

For any $\left(x_{g}, y_{g}, \theta_{g}\right) \in G$ the action of $G$ on the state space $\mathbb{R}^{2} \times S^{1}$ corresponds to multiplication "."

$\varphi_{\left(x_{g}, y_{g}, \theta_{g}\right)}(x, y, \theta)=\left(\begin{array}{l}x_{g} \\ y_{g} \\ \theta_{g}\end{array}\right) \cdot\left(\begin{array}{l}x \\ y \\ \theta\end{array}\right), \psi_{\left(x_{g}, y_{g}, \theta_{g}\right)}(u, v)=\left(\begin{array}{l}u \\ v\end{array}\right)$.

The dynamics is indeed invariant in the sense of definition 2. Take $\left(x_{g}, y_{g}, \theta_{g}\right) \in G$ and $(x, y, \theta) \in \mathbb{R}^{2} \times S^{1}$ and $(u, v) \in \mathcal{U}=\mathbb{R}^{2}$. Set $\varphi_{\left(x_{g}, y_{g}, \theta_{g}\right)}(x, y, \theta)=(X, Y, \Theta)$ and 
$\psi_{\left(x_{g}, y_{g}, \theta_{g}\right)}(u, v)=(U, V)$ (transformation (3)). The dynamics in the new variables reads the same

$$
\frac{d}{d t} X=U \cos \Theta, \quad \frac{d}{d t} Y=V \sin \Theta, \quad \frac{d}{d t} \Theta=U V .
$$

The output function is equivariant in the sense of definition 3 since for any $x_{g}, y_{g}, \theta_{g}, x$ and $y$ we have $\varrho_{\left(x_{g}, y_{g}, \theta_{g}\right)}(x, y)=$ $\left(\begin{array}{l}x \cos \theta_{g}-y \sin \theta_{g}+x_{g} \\ x \sin \theta_{g}+y \cos \theta_{g}+y_{g}\end{array}\right)$. We apply method of Section II-D to build an invariant pre-observer.

a) Invariant output error: The normalization equations (8) write with $c=0$

$$
\begin{array}{r}
x \cos \theta_{\gamma}-y \sin \theta_{\gamma}+x_{\gamma}=0 \\
x \sin \theta_{\gamma}+y \cos \theta_{\gamma}+y_{\gamma}=0 \\
\theta+\theta_{\gamma}=0
\end{array}
$$

hence

$$
\left(\begin{array}{l}
x_{\gamma} \\
y_{\gamma} \\
\theta_{\gamma}
\end{array}\right)=\left(\begin{array}{l}
x \\
y \\
\theta
\end{array}\right)^{-1}=\left(\begin{array}{c}
-x \cos \theta-y \sin \theta \\
x \sin \theta-y \cos \theta \\
-\theta
\end{array}\right)=\gamma\left(\begin{array}{l}
x \\
y \\
\theta
\end{array}\right) .
$$

A complete set of invariants is given by [see (9)]: $I(x, y, \theta, u, v)=\psi_{\gamma(x, y, \theta)}(u, v)=\left(\begin{array}{l}u \\ v\end{array}\right)$. Let $\left(x_{\gamma}, y_{\gamma}, \theta\right)^{T}=\gamma(\hat{x}, \hat{y}, \hat{\theta})^{T}$. An invariant output error writes [see (11)]

$$
\begin{aligned}
E & =\varrho_{\left(x_{\gamma}, y_{\gamma}, \theta_{\gamma}\right)}(\hat{x}, \hat{y})-\varrho_{\left(x_{\gamma}, y_{\gamma}, \theta_{\gamma}\right)}(x, y) \\
& =\left(\begin{array}{cc}
\cos \theta_{\gamma} & -\sin \theta_{\gamma} \\
\sin \theta_{\gamma} & \cos \theta_{\gamma}
\end{array}\right)\left(\begin{array}{c}
\hat{x}-x \\
\hat{y}-y
\end{array}\right)+\left(\begin{array}{l}
x_{\gamma} \\
y_{\gamma}
\end{array}\right)-\left(\begin{array}{c}
x_{\gamma} \\
y_{\gamma}
\end{array}\right) \\
& =\left(\begin{array}{cc}
\cos \hat{\theta} & \sin \hat{\theta} \\
-\sin \hat{\theta} & \cos \hat{\theta}
\end{array}\right)\left(\begin{array}{c}
\hat{x}-x \\
\hat{y}-y
\end{array}\right) .
\end{aligned}
$$

b) Invariant frame: To build an invariant frame we apply formula (5). Since $\left(D \varphi_{\gamma(x, y, \theta)}(x, y, \theta)\right)^{-1}=$ $D \varphi_{\gamma^{-1}(x, y, \theta)}(x, y, \theta)$ and here $\gamma^{-1}(x, y, \theta)=(x, y, \theta)$ an invariant frame $\left(w_{1}, w_{2}, w_{3}\right)$ is given by the image of the canonical basis of $\mathbb{R}^{2} \times \mathbb{S}^{1}$ by $D \varphi_{(x, y, \theta)}$, i.e, $\left(w_{1} w_{2} w_{3}\right)$ are the columns of the matrix

$W=D \varphi_{(x, y, \theta)}(x, y, \theta)\left(\begin{array}{lll}1 & 0 & 0 \\ 0 & 1 & 0 \\ 0 & 0 & 1\end{array}\right)=\left(\begin{array}{ccc}\cos \theta & -\sin \theta & 0 \\ \sin \theta & \cos \theta & 0 \\ 0 & 0 & 1\end{array}\right)$

and we note that it corresponds to the Frenet frame.

c) Invariant pre-observer: Any invariant pre-observer reads [see (7)]

$$
\begin{aligned}
& \frac{d}{d t}\left(\begin{array}{c}
\hat{x} \\
\hat{y} \\
\hat{\theta}
\end{array}\right)=\left(\begin{array}{c}
u \cos \hat{\theta} \\
u \sin \hat{\theta} \\
u v
\end{array}\right) \\
& +\left(\begin{array}{ccc}
\cos \hat{\theta} & -\sin \hat{\theta} & 0 \\
\sin \hat{\theta} & \cos \hat{\theta} & 0 \\
0 & 0 & 1
\end{array}\right) \overline{\mathcal{L}} \times\left(\begin{array}{cc}
\cos \hat{\theta} & \sin \hat{\theta} \\
-\sin \hat{\theta} & \cos \hat{\theta}
\end{array}\right)\left(\begin{array}{c}
\hat{x}-x \\
\hat{y}-y
\end{array}\right)
\end{aligned}
$$

where $\overline{\mathcal{L}}$ is a smooth $3 \times 2$ gain matrix whose entries depend on the invariant error $E$ but also on the invariants $I(\hat{x}, \hat{y}, \hat{\theta}, u, v)$. d) Error equation: The variable we choose to make the normalization with is $(\hat{x}, \hat{y}, \hat{\theta})^{T}$. The invariant state-error thus reads (see Section II-F)

$$
\begin{aligned}
\eta & =\gamma(\hat{x}, \hat{y}, \hat{\theta}) \cdot\left(\begin{array}{c}
\hat{x} \\
\hat{y} \\
\hat{\theta}
\end{array}\right)-\gamma(\hat{x}, \hat{y}, \hat{\theta}) \cdot\left(\begin{array}{c}
x \\
y \\
\theta
\end{array}\right) \\
& =-\left(\begin{array}{c}
\hat{x} \\
\hat{y} \\
\hat{\theta}
\end{array}\right) \cdot\left(\begin{array}{c}
x \\
y \\
\theta
\end{array}\right)=\left(\begin{array}{c}
(\hat{x}-x) \cos \hat{\theta}+(\hat{y}-y) \sin \hat{\theta} \\
-(\hat{x}-x) \sin \hat{\theta}+(\hat{y}-y) \cos \hat{\theta} \\
(\hat{\theta}-\theta)
\end{array}\right)
\end{aligned}
$$

and let us denote by $\eta=\left(\eta_{x}, \eta_{y}, \eta_{\theta}\right)^{T}$ its coordinates in $\mathbb{R}^{2} \times \mathbb{S}^{1}$. Note the first two coordinates of the state error coincide with the invariant output error: $\left(\eta_{x}, \eta_{y}\right)=\left(E_{x}, E_{y}\right)$. Let $c \theta$ denote $\cos \theta$ and $s \theta=\sin \theta$. Direct computations based on

$$
\begin{aligned}
\text { - } & \left(\begin{array}{l}
\eta_{x} \\
\eta_{y}
\end{array}\right)=\left(\begin{array}{cc}
c \hat{\theta} & s \hat{\theta} \\
-s \hat{\theta} & c \hat{\theta}
\end{array}\right)\left(\begin{array}{c}
\hat{x}-x \\
\hat{y}-y
\end{array}\right) ; \\
\text { - } & (d / d t)\left(\begin{array}{cc}
c \hat{\theta} & s \hat{\theta} \\
-s \hat{\theta} & c \hat{\theta}
\end{array}\right) \\
= & \left(u v+\overline{\mathcal{L}}_{31} \eta_{x}+\overline{\mathcal{L}}_{32} \eta_{y}\right)\left(\begin{array}{cc}
c(\hat{\theta}+\pi / 2) & s(\hat{\theta}+\pi / 2) \\
-s(\hat{\theta}+\pi / 2) & c(\hat{\theta}+\pi / 2)
\end{array}\right) ; \\
\text { - } & (d / d t)\left(\begin{array}{c}
\hat{x}-x \\
\hat{y}-y \\
\hat{\theta}-\theta
\end{array}\right) \\
& \left(\begin{array}{c}
u(c \hat{\theta}-c \theta) \\
u(s \hat{\theta}-s \theta) \\
0
\end{array}\right)+\left(\begin{array}{ccc}
c \hat{\theta} & -s \hat{\theta} & 0 \\
s \hat{\theta} & c \hat{\theta} & 0 \\
0 & 0 & 1
\end{array}\right) \overline{\mathcal{L}}\left(\begin{array}{l}
\eta_{x} \\
\eta_{y}
\end{array}\right) ;
\end{aligned}
$$

yield the following autonomous error equation:

$$
\begin{gathered}
\frac{d}{d t}\left(\begin{array}{l}
\eta_{x} \\
\eta_{y} \\
\eta_{\theta}
\end{array}\right)=\left(\begin{array}{c}
u\left(1-\cos \eta_{\theta}\right)+\left(u v+\overline{\mathcal{L}}_{31} \eta_{x}+\overline{\mathcal{L}}_{32} \eta_{y}\right) \eta_{y} \\
u \sin \eta_{\theta}-\left(u v+\overline{\mathcal{L}}_{31} \eta_{x}+\overline{\mathcal{L}}_{32} \eta_{y}\right) \eta_{x} \\
0
\end{array}\right) \\
+\overline{\mathcal{L}}\left(\begin{array}{c}
\eta_{x} \\
\eta_{y}
\end{array}\right) .
\end{gathered}
$$

Indeed the invariant error equation is independent of the trajectory and only depends on the relative quantities $\eta_{x}, \eta_{y}$ and $\eta_{\theta}$ as predicted by theorem 3 since here the invariants $I$ are $(u, v)$.

e) Convergence of the Error System: We can here tune the gains so that the error system is almost globally asymptotically convergent, i.e., it converges for any initial condition except one. The error equation writes

$$
\begin{aligned}
\frac{d}{d t} \eta_{x}= & u\left(1-\cos \eta_{\theta}\right)+\left(u v+\overline{\mathcal{L}}_{31} \eta_{x}+\overline{\mathcal{L}}_{32} \eta_{y}\right) \eta_{y} \\
& +\overline{\mathcal{L}}_{11} \eta_{x}+\overline{\mathcal{L}}_{12} \eta_{y} \\
\frac{d}{d t} \eta_{y}= & u \sin \eta_{\theta}-\left(u v+\overline{\mathcal{L}}_{31} \eta_{x}+\overline{\mathcal{L}}_{32} \eta_{y}\right) \eta_{x}+\overline{\mathcal{L}}_{21} \eta_{x}+\overline{\mathcal{L}}_{22} \eta_{y} \\
\frac{d}{d t} \eta_{\theta}= & \overline{\mathcal{L}}_{31} \eta_{x}+\overline{\mathcal{L}}_{32} \eta_{y} .
\end{aligned}
$$

Take

$$
\overline{\mathcal{L}}=\left(\begin{array}{cc}
-|u| a & 0 \\
0 & -|u| c \\
0 & -u b
\end{array}\right)+\left(\begin{array}{cc}
0 & u b E_{y}-u v \\
u v-u b E_{y} & 0 \\
0 & 0
\end{array}\right)
$$


where, $a, b, c$ are positive scalar constants, recalling that $E_{y}=$ $\eta_{y}$ the error equation is

$$
\begin{aligned}
& \frac{d}{d t} \eta_{x}=u\left(1-\cos \eta_{\theta}\right)-|u| a \eta_{x} \\
& \frac{d}{d t} \eta_{y}=u \sin \eta_{\theta}-|u| c \eta_{y} \\
& \frac{d}{d t} \eta_{\theta}=-u b \eta_{y} .
\end{aligned}
$$

Let us suppose $\int_{t_{0}}^{\infty}|u(t)| d t=+\infty$ for all $t_{0}>0$. Consider the regular change of time scale: $d s=|u| d t$, we have $\left(\epsilon_{1}= \pm 1\right.$ is the sign of $u$ )

$$
\begin{aligned}
& \frac{d}{d s} \eta_{x}=\epsilon_{1}\left(1-\cos \eta_{\theta}\right)-a \eta_{x} \\
& \frac{d}{d s} \eta_{y}=\epsilon_{1} \sin \eta_{\theta}-c \eta_{y}, \quad \frac{d}{d s} \eta_{\theta}=-\epsilon_{1} b \eta_{y}
\end{aligned}
$$

with the following triangular structure:

$$
\begin{aligned}
\frac{d^{2}}{d s^{2}} \eta_{\theta} & =-c \frac{d}{d s} \eta_{\theta}-b \sin \eta_{\theta} \\
\frac{d}{d s} \eta_{x} & =\epsilon_{1}\left(1-\cos \eta_{\theta}\right)-a \eta_{x}
\end{aligned}
$$

The first equation is the dynamics of the damped non-linear pendulum with the almost globally stable equilibrium $\eta_{\theta}=0$. The second equation is just a first order stable linear system with $\epsilon_{1}\left(1-\cos \eta_{\theta}\right)$ as source term. We proved.

Theorem 4: Consider the system (15). Assume $\int_{t_{0}}^{\infty}|u(t)| d t=+\infty$ for all $t_{0}>0$. The non-linear observer

$$
\begin{aligned}
& \frac{d}{d t}\left(\begin{array}{c}
\hat{x} \\
\hat{y} \\
\hat{\theta}
\end{array}\right)=\left(\begin{array}{c}
u \cos \hat{\theta} \\
u \sin \hat{\theta} \\
u v
\end{array}\right) \\
& \quad+\left(\begin{array}{ccc}
\cos \hat{\theta} & -\sin \hat{\theta} & 0 \\
\sin \hat{\theta} & \cos \hat{\theta} & 0 \\
0 & 0 & 1
\end{array}\right) \overline{\mathcal{L}}\left(\begin{array}{cc}
\cos \hat{\theta} & \sin \hat{\theta} \\
-\sin \hat{\theta} & \cos \hat{\theta}
\end{array}\right)\left(\begin{array}{c}
\hat{x}-x \\
\hat{y}-y
\end{array}\right)
\end{aligned}
$$

with

$$
\overline{\mathcal{L}}=\left(\begin{array}{cc}
-|u| a & 0 \\
0 & -|u| c \\
0 & -u b
\end{array}\right)+\left(\begin{array}{cc}
0 & u b E_{y}-u v \\
u v-u b E_{y} & 0 \\
0 & 0
\end{array}\right)
$$

is almost globally asymptotically convergent.

\section{B. A Chemical Reactor}

We consider the classical exothermic reactor of [4]. With slightly different notations, the dynamics reads

$$
\begin{aligned}
\frac{d}{d t} X^{i n} & =0 \\
\frac{d}{d t} X & =D(t)\left(X^{i n}-X\right)-k \exp \left(-\frac{E_{A}}{R T}\right) X \\
\frac{d}{d t} T & =D(t)\left(T^{i n}(t)-T\right)+c \exp \left(-\frac{E_{A}}{R T}\right) X+v(t) \\
y & =T
\end{aligned}
$$

where $\left(E_{A}, R, k, c\right)$ are positive and known constant parameters, $D(t), T^{i n}(t)$ and $v(t)$ are known time functions and $D(t) \geq 0$. The available online measurement is $T$ : the temperature inside the reactor. The parameter $X^{i n}>0$, the inlet composition, is unknown. The reactor composition $X$ is not measured.

These two differential equations correspond to material and energy balances. Their structure is independent of the units: the equations write the same whether they are written in $\mathrm{mol} / \mathrm{l}$ or in $\mathrm{kg} / \mathrm{l}$ for instance. Let us formalize such independence in terms of invariance. We just consider a change of material unit corresponding to the following scaling $X \mapsto g X$ and $X^{i n} \mapsto g X^{i n}$ with $g>0$. The group $G$ is the multiplicative group $\mathbb{R}_{+}^{*}$. Take $x=\left(X^{i n}, X, T\right)$ as state and $u=\left(c, D(t), T^{i n}(t), v(t)\right)$ as known input. The action on $\mathcal{X} \times \mathcal{U}$ is defined for each $g>0$ via the (linear) transformations

$$
\varphi_{g}(x)=\left(\begin{array}{c}
g X^{i n} \\
g X \\
T
\end{array}\right), \quad \psi_{g}(u)=\left(\begin{array}{c}
c / g \\
D \\
T^{i n} \\
v
\end{array}\right)
$$

The dynamics (20) is invariant in the sense of definition 2. Since $y=T$ is unchanged by $G\left(\varrho_{g}(y) \equiv y\right.$ here $)$, it is a $G$-equivariant output in the sense of definition 3 . We apply method of Section II-D to build an invariant pre-observer.

a) Invariant output error and complete set of invariants: We choose the second component of $\varphi_{g}$ for the normalization and take as normalizing equation (8): $g X=1$, i.e. $\gamma(x)=$ $1 / X$. Then using (11) the invariant output error is $E(\hat{x}, u, y)=$ $\hat{T}-y$ and using (9) the complete set of invariant $I$ is made of the remaining components of $\varphi_{1 / \hat{x}}(\hat{x})$ and $\psi_{1 / \hat{x}}(u): I(\hat{x}, u)=$ $\left(\hat{x}^{i n} / \hat{x}, \hat{T}, c \hat{x}, D, T^{i n}, v\right)$.

b) Invariant frame: According to (5), an invariant frame is

$$
w_{1}=X^{i n} \frac{\partial}{\partial X^{i n}}, \quad w_{2}=X \frac{\partial}{\partial X}, \quad w_{3}=\frac{\partial}{\partial T}
$$

where $w_{1}$ has been multiplied by the scalar invariant $X^{i n} / X$.

c) Invariant pre-observer: According to theorem 1, invariant pre-observers have the following structure:

$$
\left\{\begin{array}{l}
\frac{d}{d t} \hat{X}^{i n}=\mathcal{L}_{1}(I(\hat{x}, u), \hat{T}-T) \hat{X}^{i n} \\
\frac{d}{d t} \hat{X}=D(t)\left(\hat{X}^{i n}-\hat{X}\right)-k \exp \left(-\frac{E_{A}}{R \hat{T}}\right) \hat{X} \\
\quad+\mathcal{L}_{2}(I(\hat{x}, u), \hat{T}-T) \hat{X} \\
\frac{d}{d t} \hat{T}=D(t)\left(T^{i n}(t)-\hat{T}\right)+c \exp \left(-\frac{E_{A}}{R \hat{T}}\right) \hat{X}+v(t) \\
\quad+\mathcal{L}_{3}(I(\hat{x}, u), \hat{T}-T)
\end{array}\right.
$$

where the $\mathcal{L}_{i}$ 's are smooth scalar functions such that $\mathcal{L}_{i}(I, 0) \equiv$ 0 . We have the desirable property that any invariant observer preserves the fact that $\hat{X}$ and $\hat{X}^{i n}$ are positive quantities. Indeed the domain $\left\{\left(\hat{X}^{i n}, \hat{X}, T\right) \in \mathbb{R}^{3} \mid \hat{X}^{i n}>0, \hat{X}>0\right\}$ is positively invariant for (21), whatever the choices made for $\mathcal{L}_{1}, \mathcal{L}_{2}$ and $\mathcal{L}_{3}$ $(D(t) \geq 0)$. 
d) Convergence around an equilibrium: Assume that around a steady-state $\left(\bar{X}^{i n}, \bar{X}, \bar{T}\right)$ of (20), we designed the three constant gains $L_{1}, L_{2}$, and $L_{3}$, such that

$$
\begin{aligned}
\frac{d}{d t} \hat{X}^{i n} & =L_{1}(\hat{T}-T) \\
\frac{d}{d t} \hat{X} & =D(t)\left(\hat{X}^{i n}-\hat{X}\right)-k \exp \left(-\frac{E_{A}}{R \hat{T}}\right) \hat{X}+L_{2}(\hat{T}-T) \\
\frac{d}{d t} \hat{T}= & D(t)\left(T^{i n}(t)-\hat{T}\right)+c \exp \left(-\frac{E_{A}}{R \hat{T}}\right) \hat{X} \\
& +v(t)+L_{3}(\hat{T}-T)
\end{aligned}
$$

is locally convergent around $\left(\bar{X}^{i n}, \bar{X}, \bar{T}\right)$. Then following the procedure of Section II-E, we get the invariant observer:

$$
\begin{aligned}
\frac{d}{d t} \hat{X}^{i n}= & L_{1}(\hat{T}-T) \frac{\hat{X}^{i n}}{\bar{X}^{i n}} \\
\frac{d}{d t} \hat{X}= & D(t)\left(\hat{X}^{i n}-\hat{X}\right)-k \exp \left(-\frac{E_{A}}{R \hat{T}}\right) \hat{X} \\
& +L_{2}(\hat{T}-T) \frac{\hat{X}}{\bar{X}} \\
\frac{d}{d t} \hat{T}= & D(t)\left(T^{i n}(t)-\hat{T}\right)+c \exp \left(-\frac{E_{A}}{R \hat{T}}\right) \hat{X} \\
& +v(t)+L_{3}(\hat{T}-T)
\end{aligned}
$$

that exhibits identical performances around the steady-state. Moreover it provides automatically positive estimations for $X$ and $X^{i n}$, and the performances are independent of the choice of units.

e) Invariant error and global convergence of the observer: As the dimension of $G$ is strictly smaller than the dimension of $\mathcal{X}$ it is interesting to use the base and fiber coordinates of Section II-B-4 which are in this case globally defined on the physical domain $\left\{\left(X^{i n}, X, T\right) \in \mathbb{R}^{3} \mid X^{i n}>0, X>0\right\}$. Consider the following change of variable:

$$
\left(\begin{array}{c}
X \\
X^{i n} \\
T
\end{array}\right) \mapsto\left(\begin{array}{c}
Z=\log (X) \\
\xi=\log \left(X / X^{i n}\right) \\
T
\end{array}\right) .
$$

Indeed $X$ corresponds to fiber coordinate and $X / X^{i n}, T$ to base coordinates. We took the log of these quantities so that the computation of time derivatives is easier. The dynamics (20) now writes

$$
\begin{aligned}
\frac{d}{d t} Z & =D(\exp (-\xi)-1)-k \exp \left(-\frac{E_{A}}{R T}\right) \\
\frac{d}{d t} \xi & =D(\exp (-\xi)-1)-k \exp \left(-\frac{E_{A}}{R T}\right) \\
\frac{d}{d t} T & =D\left(T^{i n}-T\right)+c \exp Z \exp \left(-\frac{E_{A}}{R T}\right)+v(t)
\end{aligned}
$$

and the invariant observer (21) writes

$$
\begin{aligned}
\frac{d}{d t} \hat{Z} & =D(\exp (-\hat{\xi})-1)-k \exp \left(-\frac{E_{A}}{R \hat{T}}\right)+\mathcal{L}_{2} \\
\frac{d}{d t} \hat{\xi} & =D(\exp (-\hat{\xi})-1)-k \exp \left(-\frac{E_{A}}{R \hat{T}}\right)+\mathcal{L}_{2}-\mathcal{L}_{1} \\
\frac{d}{d t} \hat{T} & =D\left(T^{i n}-\hat{T}\right)+c \exp \hat{Z} \exp \left(-\frac{E_{A}}{R \hat{T}}\right)+v(t)+\mathcal{L}_{3} .
\end{aligned}
$$

Consider the following gain design $(\beta>0$ and $\kappa>0$ are two arbitrary parameters)

$$
\begin{aligned}
\mathcal{L}_{2}= & -\beta c \exp \hat{Z} \exp \left(-\frac{E_{A}}{R T}\right)(\hat{T}-T) \\
& +k \exp \left(-\frac{E_{A}}{R \hat{T}}\right)-k \exp \left(-\frac{E_{A}}{R T}\right) \\
\mathcal{L}_{2}-\mathcal{L}_{1}= & k \exp \left(-\frac{E_{A}}{R \hat{T}}\right)-k \exp \left(-\frac{E_{A}}{R T}\right) \\
\mathcal{L}_{3}= & \left(-\kappa c \exp \left(-\frac{E_{A}}{R T}\right)(\hat{T}-T)\right. \\
& \left.-c \exp \left(-\frac{E_{A}}{R \hat{T}}\right)+c \exp \left(-\frac{E_{A}}{R T}\right)\right) \exp \hat{Z}+D(\hat{T}-T) .
\end{aligned}
$$

The choice of such non-linear gains ensure global asymptotic stability when there exists $M$ and $\alpha>0$ such that the measurements verify for all $t \geq 0, M \geq X^{\text {in }}, D(t), T(t) \geq \alpha$. It implies [see (20)] there exists $\sigma>0$ such that $t \geq 0, M \geq$ $X^{i n}, X(t), D(t), T(t) \geq \sigma$. The design, although specific to the example relies on the notion of invariant state error (see Section II-F). Since the normalizing equation (8) is: $g X=1$, i.e. $\gamma(x)=1 / X$ the invariant state-error writes in the new variables $\eta=(\tilde{Z}, \tilde{\xi}, \tilde{T})$ where

$$
\begin{aligned}
\tilde{Z} & =\hat{Z}-Z=\log (\hat{X} / X) \\
\tilde{\xi} & =\hat{\xi}-\xi=\log \left(\hat{X} / \hat{X}^{i n}\right)-\log \left(X / X^{i n}\right) . \\
\tilde{T} & =\hat{T}-T
\end{aligned}
$$

The dynamics of the invariant state error is the following:

$$
\begin{aligned}
\frac{d}{d t} \tilde{Z}= & D(\exp (-\hat{\xi})-\exp (-\xi))-\beta c \exp \left(Z-\frac{E_{A}}{R T}\right) \exp \tilde{Z} \tilde{T} \\
\frac{d}{d t} \tilde{\xi}= & D(\exp (-\hat{\xi})-\exp (-\xi)) \\
\frac{d}{d t} \tilde{T}= & c \exp \left(Z-\frac{E_{A}}{R T}\right)(\exp \tilde{Z}-1) \\
& -\kappa c \exp \left(Z-\frac{E_{A}}{R T}\right) \exp \tilde{Z} \tilde{T} .
\end{aligned}
$$

Since $M \geq D(t) \geq \sigma$, we have $\lim _{t \mapsto+\infty}(\hat{\xi}(t)-\xi(t))=0$, which means the dynamics of the system on the base coordinate $\xi$ converges independently from its initial value. And the system writes

$$
\begin{aligned}
\frac{d}{d t} \tilde{Z}= & -\beta c \exp \left(-\frac{E_{A}}{R T}+Z\right) \exp \tilde{Z} \tilde{T}+\epsilon_{1}(t) \\
\frac{d}{d t} \tilde{T}= & c \exp \left(-\frac{E_{A}}{R T}+Z\right)(\exp \tilde{Z}-1) \\
& -\kappa c \exp \left(-\frac{E_{A}}{R T(t)}+Z\right) \exp \tilde{Z} \tilde{T}
\end{aligned}
$$

where $\epsilon_{1}(t)=D(\exp (-\hat{\xi}(t))-\exp (-\xi(t)))$ and we know that $\lim _{t \mapsto+\infty} \epsilon_{1}(t)=0$ and $\int\left|\epsilon_{1}(t)\right| d t<\infty$. Consider the regular change of time scale $\tau=\int_{0}^{t} c \exp \left(-\left(E_{A} / R T(s)\right)+Z(s)\right) d s$. Then:

$$
\begin{aligned}
& \frac{d \tilde{Z}}{d \tau}=-\beta \exp \tilde{Z} \tilde{T}+\epsilon(t) \\
& \frac{d \tilde{T}}{d \tau}=(\exp \tilde{Z}-1)-\kappa \exp \tilde{Z} \tilde{T}
\end{aligned}
$$


where $\epsilon(t)=c X \exp \left(\left(E_{A} / R T\right)-Z\right) \epsilon_{1}(t)$. Take $V=$ $\tilde{Z}+\exp (-\tilde{Z})+(\beta / 2) \tilde{T}^{2}$ as Lyapounov function. $(d / d t) V \leq$ $|\epsilon(t)|(1+2 V)$. Thus $V$ is bounded and so are the trajectories. Let $(\bar{Z}(t), \bar{T}(t))$ be a trajectory. Take $U(t)=V(\bar{Z}, \bar{T}, t)-$ $\int_{t}^{\infty} \epsilon(\tau)\left(1-\exp (-\bar{Z}(\tau)) d \tau .(d / d t) U=-\beta \kappa \bar{T}^{2} \exp \bar{Z}<0\right.$. A standard application of Barbalat's lemma shows that $(0,0)$ is globally asymptotically stable. We proved.

Theorem 5: Consider the system (20). Assume there exist $M$ and $\alpha>0$ such that for all $t \geq 0, M \geq X^{i n}, D(t), T(t) \geq \alpha$. Then for any $\beta, \kappa>0$ the following non-linear observer:

$$
\begin{aligned}
\frac{d}{d t} \hat{X}^{i n}= & -\beta \exp \left(-\frac{E_{A}}{R T(t)}\right)(\hat{T}-T(t)) c \hat{X} \hat{X}^{i n} \\
\frac{d}{d t} \hat{X}= & D(t)\left(\hat{X}^{i n}-\hat{X}\right)-\exp \left(-\frac{E_{A}}{R T(t)}\right) \\
& \times(k+\beta(\hat{T}-T(t)) c \hat{X}) \hat{X} \\
\frac{d}{d t} \hat{T}= & \exp \left(-\frac{E_{A}}{R T(t)}\right)(1-\kappa(\hat{T}-T(t))) c \hat{X} \\
& +D(t)\left(T^{i n}(t)-T(t)\right)+v(t)
\end{aligned}
$$

is globally converging.

\section{Velocity-Aided Inertial Navigation}

In low-cost navigation systems, the relatively inaccurate gyroscopes and accelerometers are "aided" by velocity measurements (given by an air-data system or a Doppler radar) and magnetic sensors. The various measurements are then "merged" according to the (flat-Earth) motion equations of the aircraft, usually by a gain-scheduled observer or an extended Kalman filter. The convergence analysis, hence the tuning, of such an observer is far from easy. Using our theory, we derive in this section a simple invariant observer, which yields an error equation independent of the trajectory of the aircraft. The tuning of the gains to achieve local convergence around any trajectories is thus straightforward.

Simulations illustrate the good behavior of the observer even in the presence of noise and sensor biases. They moreover indicate that the domain of convergence of the observer with respect to the initial condition should be very large (though we have not investigated the global behavior).

The derivation of the observer and its implementation are strongly simplified when the body orientation is described by a quaternion of length 1 (rather than by Euler angles or a rotation matrix).

1) Quaternions: As in [9], we use the quaternion parametrization of $\mathrm{SO}(3)$ to derive filters for state estimation. The quaternions are a non commutative group. Any quaternion $q$ can be written $q=q^{0}+q^{1} e_{1}+q^{2} e_{2}+q^{3} e_{3}$ with $\left(q^{0}, q^{1}, q^{2}, q^{3}\right) \in \mathbb{R}^{4}$, the multiplication $*$ is defined by $e_{1} * e_{1}=-1, e_{1} * e_{2}=-e_{2} * e_{1}=e_{3}$ and circular permutations.

The norm of $q$ is $\sqrt{\left(q^{0}\right)^{2}+\left(q^{1}\right)^{2}+\left(q^{2}\right)^{2}+\left(q^{3}\right)^{2}}$. Any vector $\vec{p} \in \mathbb{R}^{3}$ can be identified with the quaternion $p^{1} e_{1}+p^{2} e_{2}+p^{3} e_{3}$. We will make this identification systematically. Then one can associate to any quaternion whose norm is 1 , a rotation matrix $R_{q} \in S O(3)$ thanks to the following relation: $q^{-1} * \vec{p} * q=$ $R_{q} \vec{p}$ for all $\vec{p}$. The subgroup of quaternions whose norm is 1 is denoted by $\mathbb{H}_{1}$. Conversely, to any rotation $R_{q}$ of $S O(3)$ are associated two quaternions $\pm q$ of length 1 . Thus although the state space in the example is $S O(3) \times \mathbb{R}^{3}$, we will write the elements of $S O(3)$ as quaternions whose norm is 1 (denoted by $\mathbb{H}_{1}$ ) and the vectors of $\mathbb{R}^{3}$ as quaternions whose first coordinate is equal to 0 . Numerically, quaternions are easier to manipulate and to compute than matrices in $S O(3)$. The wedge product $v \times \omega$ of vectors of $\mathbb{R}^{3}$ writes for the associated quaternions: $(v * \omega-\omega * v) / 2$.

2) Motion Equations: The motion of a flying rigid body (assuming the Earth is flat and defines an inertial frame) is described by

$$
\begin{aligned}
\frac{d}{d t} q & =\frac{1}{2} q * \omega \\
\frac{d}{d t} v & =v \times \omega+q^{-1} * \mathbf{A}_{g r} * q+a \\
y & =\left(y_{v}, y_{b}\right)=\left(v, q^{-1} * \mathbf{B} * q\right)
\end{aligned}
$$

where $\left(\omega, a, \mathbf{A}_{g r}, \mathbf{B}\right)$ are inputs and

- $q$ is the quaternion of norm 1 representing the orientation of the body-fixed frame with respect to the earth-fixed frame. Notice the norm of $q$ is left unchanged by the first equation because $\omega$ is a vector of $\mathbb{R}^{3}$ (i.e. a quaternion whose first coordinate is 0 ).

- $\omega=\omega^{1} e_{1}+\omega^{2} e_{2}+\omega^{3} e_{3}$ is the instantaneous angular velocity vector in the body-fixed frame.

- $v=v^{1} e_{1}+v^{2} e_{2}+v^{3} e_{3}$ is the velocity vector of the center of mass in the body-fixed frame

- $\mathbf{A}_{g r}=\mathbf{A}_{g r}^{1} e_{1}+\mathbf{A}_{g r}^{2} e_{2}+\mathbf{A}_{g r}^{3} e_{3}$ is the gravity vector in the earth-fixed frame.

- $a=a^{1} e_{1}+a^{2} e_{2}+a^{3} e_{3}$ is the specific acceleration vector, i.e, the aerodynamics forces divided by the body mass.

- $\mathbf{B}=\mathbf{B}^{1} e_{1}+\mathbf{B}^{2} e_{2}+\mathbf{B}^{3} e_{3}$ is the earth's magnetic field expressed in the earth-fixed frame.

$\mathbf{A}_{g r}$ and $\mathbf{B}$ are constant over the flying area. The first equation describes the kinematics of the body, the second is Newton's force law. The measurements are $\omega(t), a(t), v(t)$ and $q^{-1}(t) * \mathbf{B} * q(t)$ (measured by gyroscopes, accelerometers, air data system or Doppler radar and magnetic sensors). Their coordinates are known in the body-fixed frame. The goal is to estimate $q$ (which is not measured) and $v$ (the noisy measurement $v(t)$ must be filtered).

3) Invariance of the Motion Equations: From physical consideration, the symmetries are associated to the group $G=$ $S E(3)$ which consists of rotations and translations in dimension 3 . We identify (up to the multiplication group $\{-1,+1\}$ ) $G$ and the state space $\mathcal{X}=\mathbb{H}_{1} \times \mathbb{R}^{3}$. For any $\left(q_{g}, v_{g}\right) \in G$, the $\operatorname{map} \varphi_{\left(q_{g}, v_{g}\right)}$ corresponds to the following group multiplication on $G$ :

$$
\varphi_{\left(q_{g}, v_{g}\right)}(q, v)=\left(\begin{array}{l}
q_{g} \\
v_{g}
\end{array}\right) \cdot\left(\begin{array}{l}
q \\
v
\end{array}\right)=\left(\begin{array}{c}
q * q_{g} \\
q_{g}^{-1} * v * q_{g}+v_{g} .
\end{array}\right)
$$

Here $u=\left(\begin{array}{c}a \\ \omega\end{array}\right)$. And the map $\psi_{\left(q_{g}, v_{g}\right)}$ is given by

$$
\psi_{\left(q_{g}, v_{g}\right)}(a, \omega)=\left(\begin{array}{c}
q_{g}^{-1} * a * q_{g}-v_{g} \times\left(q_{g}^{-1} * \omega * q_{g}\right) \\
q_{g}^{-1} * \omega * q_{g}
\end{array}\right) .
$$

Take $\left(q_{g}, v_{g}\right) \in G,(q, v) \in G$ and $(a, \omega) \in \mathcal{U}$. Set [transformation (3)] $\varphi_{\left(q_{g}, v_{g}\right)}(q, v)=(Q, V)$ and $\psi_{\left(q_{g}, v_{g}\right)}(a, \omega)=(A, \Omega)$. We have

$$
\frac{d}{d t} Q=\frac{1}{2} q * \omega * q_{g}=\frac{1}{2} q * q_{g} * q_{g}^{-1} * \omega * q_{g}=\frac{1}{2} Q * \Omega
$$


and one can check $(d / d t) V=V \times \Omega+Q^{-1} * \mathbf{A}_{g r} * Q+A$. Thus the dynamics in the new variables reads the same: it is invariant in the sense of definition 2. The output function

$$
y=\left(y_{v}, y_{b}\right)=\left(v, q^{-1} * \mathbf{B} * q\right)
$$

is $G$-equivariant in the sense of definition 3 with

$$
\left(Y_{v}, Y_{b}\right)=\varrho_{\left(q_{g}, v_{g}\right)}\left(y_{v}, y_{b}\right)=\left(q_{g}^{-1} * y_{v} * q_{g}+v_{g}, q_{g}^{-1} * y_{b} * q_{g}\right) .
$$

4) An Invariant Pre-Observer: We apply method of Section II-D to build an invariant pre-observer.

a) Invariant output error and complete set of invariants: The normalization equations (8) write

$$
\begin{array}{r}
q * q_{\gamma}=1 \\
q_{\gamma}^{-1} * v * q_{\gamma}+v_{\gamma}=0
\end{array}
$$

(where 1 is the unit quaternion: $1+0 e_{1}+0 e_{2}+0 e_{3}$ ), hence

$$
\left(\begin{array}{l}
q_{\gamma} \\
v_{\gamma}
\end{array}\right)=\left(\begin{array}{l}
q \\
v
\end{array}\right)^{-1}=\left(\begin{array}{c}
q^{-1} \\
-q * v * q^{-1}
\end{array}\right)=\gamma\left(\begin{array}{l}
q \\
v
\end{array}\right)
$$

Using (9), a complete set of invariants is given by

$$
I(q, v, a, \omega)=\psi_{\gamma(q, v)}\left(\begin{array}{l}
\omega \\
a
\end{array}\right)=\left(\begin{array}{c}
q * \omega * q^{-1} \\
q *(a+v \times \omega) * q^{-1} .
\end{array}\right)
$$

Let $\left(q_{\gamma}, v_{\gamma}\right)$ be $\gamma(\hat{q}, \hat{v})$. Using (11), an invariant output error is given by

$$
E=\varrho_{\left(q_{\gamma}, v_{\gamma}\right)}(\hat{y})-\varrho_{\left(q_{\gamma}, v_{\gamma}\right)}(y)=\left(\begin{array}{l}
\hat{q} *(\hat{v}-v) * \hat{q}^{-1} \\
\mathbf{B}-\hat{q} * y_{b} * \hat{q}^{-1} .
\end{array}\right)
$$

b) Invariant frame: In order to make a (local) invariant frame we must take a basis of the tangent space to the identity element (although the theory is local, the frame is in fact globally defined here). The tangent space to the space of quaternions whose norm is 1 is the 3-D set of all quaternions whose first coordinate is equal to 0 . Let $e_{1}, e_{2}, e_{3}$ be the canonical basis of that space, which can be identified with the canonical basis of $\mathbb{R}^{3}$. We apply formula (5) with $x=(q, v)$. Since $\left(D \varphi_{\gamma(q, v)}(q, v)\right)^{-1}=D \varphi_{\gamma^{-1}(q, v)}(q, v)$ and here $\gamma^{-1}(q, v)=$ $(q, v)$, an invariant frame is given by the set of 6 vector fields whose values in $(q, v)$ are the following:

$$
\begin{aligned}
& D \varphi_{(q, v)}(q, v)\left(\begin{array}{c}
e_{i} \\
0
\end{array}\right)_{1 \leq i \leq 3}=\left(\begin{array}{c}
e_{i} * q \\
0
\end{array}\right)_{1 \leq i \leq 3} \\
& D \varphi_{(q, v)}(q, v)\left(\begin{array}{c}
0 \\
e_{i}
\end{array}\right)_{1 \leq i \leq 3}=\left(\begin{array}{c}
0 \\
q^{-1} * e_{i} * q
\end{array}\right)_{1 \leq i \leq 3} .
\end{aligned}
$$

c) Invariant pre-observer: According to theorem (1) any invariant pre-observer reads

$$
\begin{aligned}
& \frac{d}{d t} \hat{q}=\frac{1}{2} \hat{q} * \omega+\sum_{i=1}^{3} \mathcal{L}_{i}^{q}(I, E) e_{i} * \hat{q} \\
& \frac{d}{d t} \hat{v}=\hat{v} \times \omega+\hat{q}^{-1} * \mathbf{A}_{g r} * \hat{q}+a+\sum_{i=1}^{3} \mathcal{L}_{i}^{v}(I, E) \hat{q}^{-1} * e_{i} * \hat{q}
\end{aligned}
$$

where the $\mathcal{L}_{i}^{q}, \mathcal{L}_{i}^{v}$ are smooth functions of $I$ and $E$ such that $\mathcal{L}_{i}^{q}(I, 0)=0$ and $\mathcal{L}_{i}^{v}(I, 0)=0$. To put it into the alternative form (7) we decompose $E$ into $\left(E_{v}, E_{b}\right)=\left(\hat{q} *(\hat{v}-v) * \hat{q}^{-1}, \mathbf{B}-\right.$ $\left.\hat{q} * y_{b} * \hat{q}^{-1}\right)$ and write

$$
\begin{aligned}
& \mathcal{L}_{i}^{q}\left(I, E_{v}, E_{b}\right)=\overline{\mathcal{L}}_{v, i}^{q}\left(I, E_{v}, E_{b}\right) E_{v}+\overline{\mathcal{L}}_{b, i}^{q}\left(I, E_{v}, E_{b}\right) E_{b} \\
& \mathcal{L}_{i}^{v}\left(I, E_{v}, E_{b}\right)=\overline{\mathcal{L}}_{v, i}^{v}\left(I, E_{v}, E_{b}\right) E_{v}+\overline{\mathcal{L}}_{b, i}^{v}\left(I, E_{v}, E_{b}\right) E_{b}
\end{aligned}
$$

where the $\overline{\mathcal{L}}_{v, i}^{q}, \overline{\mathcal{L}}_{b, i}^{q}, \overline{\mathcal{L}}_{v, i}^{v}, \overline{\mathcal{L}}_{b, i}^{v}$ are $1 \times 3$ matrices with entries depending on $\left(I, E_{v}, E_{b}\right)$. Hence

$$
\begin{aligned}
\sum_{i=1}^{3} \mathcal{L}_{i}^{q}(I, E) e_{i} * \hat{q} & =\left(\sum_{i=1}^{3}\left(\overline{\mathcal{L}}_{v, i}^{q} E_{v}+\overline{\mathcal{L}}_{b, i}^{q} E_{b}\right) e_{i}\right) * \hat{q} \\
& =\left(\left(\begin{array}{c}
\overline{\mathcal{L}}_{v, 1}^{q} \\
\overline{\mathcal{L}}_{v, 2}^{q} \\
\overline{\mathcal{L}}_{v, 3}^{q}
\end{array}\right) E_{v}+\left(\begin{array}{c}
\overline{\mathcal{L}}_{b, 1}^{q} \\
\overline{\mathcal{L}}_{b, 2}^{q} \\
\overline{\mathcal{L}}_{b, 3}^{q}
\end{array}\right) E_{b}\right) * \hat{q}
\end{aligned}
$$

Proceeding in the same way with the other correction term $\sum_{i=1}^{3} \mathcal{L}_{i}^{v}(I, E) \hat{q}^{-1} * e_{i} * \hat{q}$, the general invariant pre-observer finally reads

$\frac{d}{d t} \hat{q}=\frac{1}{2} \hat{q} * \omega+\left(\overline{\mathcal{L}}_{v}^{q} E_{v}+\overline{\mathcal{L}}_{b}^{q} E_{b}\right) * \hat{q}$

$\frac{d}{d t} \hat{v}=\hat{v} \times \omega+\hat{q}^{-1} * \mathbf{A}_{g r} * \hat{q}+a+\hat{q}^{-1} *\left(\overline{\mathcal{L}}_{v}^{v} E_{v}+\overline{\mathcal{L}}_{b}^{v} E_{b}\right) * \hat{q}$

where $\overline{\mathcal{L}}_{v}^{q}, \overline{\mathcal{L}}_{b}^{q}, \overline{\mathcal{L}}_{v}^{v}$ and $\overline{\mathcal{L}}_{b}^{v}$, are $3 \times 3$ gain matrices whose entries depend on the invariant errors $E_{v}$ and $E_{b}$ and also on the invariants $I(\hat{q}, \hat{v}, a, \omega)$.

As a by-product of the geometric structure of the observer, we automatically have the desirable property that the norm of $\hat{q}$ is left unchanged by (23), because $\omega$ and $\overline{\mathcal{L}}_{v}^{q} E_{v}+\overline{\mathcal{L}}_{b}^{q} E_{b}$ are vectors of $\mathbb{R}^{3}$ (i.e. quaternions with a zero first coordinate).

d) Error equation: The invariant state-error as defined in Section II-F reads $\eta=\varphi_{\gamma(q, v)}(\hat{q}, \hat{v})-\varphi_{\gamma(q, v)}(q, v)$. One can write $\eta=\left(\eta_{q}, \eta_{v}\right)$ where $\eta_{q}=\hat{q} * q^{-1}-1$ and $\eta_{v}=q *(\hat{v}-$ $v) * q^{-1}$. But here the state space is viewed as the group $\operatorname{SE}(3)$ itself so we consider the equivalent state-error

$$
\eta_{q}=\hat{q} * q^{-1}, \quad \eta_{v}=q *(\hat{v}-v) * q^{-1} .
$$

so that $\eta$ is an error in the sense of group multiplication. Thus a small error corresponds to $\left(\eta_{q}, \eta_{v}\right)$ close to the group identity element $(1,0)$. Its time derivative verifies

$$
\begin{aligned}
\frac{d}{d t} \eta_{q}= & \left(\frac{1}{2} \hat{q} * \omega+\left(\overline{\mathcal{L}}_{v}^{q} E_{v}+\overline{\mathcal{L}}_{b}^{q} E_{b}\right) * \hat{q}\right) * q^{-1} \\
& -\hat{q} *\left(q^{-1} * \frac{1}{2} q * \omega * q^{-1}\right) \\
= & +\left(\overline{\mathcal{L}}_{v}^{q} E_{v}+\overline{\mathcal{L}}_{b}^{q} E_{b}\right) * \eta_{q} \\
\frac{d}{d t} \eta_{v}= & q *\left((\hat{v}-v) \times \omega+\hat{q}^{-1} * \mathbf{A}_{g r} * \hat{q}-q^{-1} * \mathbf{A}_{g r} * q\right. \\
& \left.\quad+\hat{q}^{-1} *\left(\overline{\mathcal{L}}_{v}^{v} E_{v}+\overline{\mathcal{L}}_{b}^{v} E_{b}\right) * \hat{q}\right) * q^{-1} \\
& +\frac{1}{2} q * \omega *(\hat{v}-v) * q^{-1}-q *(\hat{v}-v) * \omega * q^{-1} \\
= & q *(\hat{v}-v) \times \omega * q^{-1}+q * \omega \times(\hat{v}-v) * q^{-1} \\
& +\eta_{q}^{-1} * \mathbf{A}_{g r} * \eta_{q}-\mathbf{A}_{g r}+\eta_{q}^{-1} *\left(\overline{\mathcal{L}}_{v}^{v} E_{v}+\overline{\mathcal{L}}_{b}^{v} E_{b}\right) * \eta_{q} \\
= & \eta_{q}^{-1} * \mathbf{A}_{g r} * \eta_{q}-\mathbf{A}_{g r}+\eta_{q}^{-1} *\left(\overline{\mathcal{L}}_{v}^{v} E_{v}+\overline{\mathcal{L}}_{b}^{v} E_{b}\right) * \eta_{q}
\end{aligned}
$$


where $E_{v}=\eta_{q} * \eta_{v} * \eta_{q}^{-1}$ and $E_{b}=\mathbf{B}-\eta_{q} * \mathbf{B} * \eta_{q}^{-1}$. Thus the error system is autonomous

$$
\begin{gathered}
\frac{d}{d t} \eta_{q}=\left[\overline{\mathcal{L}}_{v}^{q}\left(\eta_{q} * \eta_{v} * \eta_{q}^{-1}\right)+\overline{\mathcal{L}}_{b}^{q}\left(\mathbf{B}-\eta_{q} * \mathbf{B} * \eta_{q}^{-1}\right)\right] * \eta_{q} \\
\frac{d}{d t} \eta_{v}=\eta_{q}^{-1} *\left[\begin{array}{r}
\mathbf{A}_{g r}+\overline{\mathcal{L}}_{v}^{v}\left(\eta_{q} * \eta_{v} * \eta_{q}^{-1}\right) \\
\left.+\overline{\mathcal{L}}_{b}^{v}\left(\mathbf{B}-\eta_{q} * \mathbf{B} * \eta_{q}^{-1}\right)\right] * \eta_{q}-\mathbf{A}_{g r}
\end{array}\right.
\end{gathered}
$$

It does depend neither on the trajectory, nor on the inputs $\omega(t)$ and $a(t)$. In the general case (see theorem 3$)(d / d t) \eta$ is a function of $\eta$ and $I$. But here it does not even depend on $I$.

e) Convergence of the linearized error system: Let us suppose $\hat{q}$ and $\hat{v}$ are close to respectively $q$ and $v$. First order approximations write $\delta E_{v}=\delta \eta_{v}$ and $\delta E_{b}=-\delta \eta_{q} * \mathbf{B}+\mathbf{B} * \delta \eta_{q}=$ $2 \mathbf{B} \times \delta \eta_{q}$. Thus the linearized error equation writes

$$
\begin{aligned}
\frac{d}{d t} \delta \eta_{q} & =\overline{\mathcal{L}}_{v}^{q} \delta \eta_{v}+2 \overline{\mathcal{L}}_{b}^{q}\left(\mathbf{B} \times \delta \eta_{q}\right) \\
\frac{d}{d t} \delta \eta_{v} & =2 \mathbf{A}_{g r} \times \delta \eta_{q}+\overline{\mathcal{L}}_{v}^{v} \delta \eta_{v}+2 \overline{\mathcal{L}}_{b}^{v}\left(\mathbf{B} \times \delta \eta_{q}\right) .
\end{aligned}
$$

Let us choose

$$
\begin{aligned}
\overline{\mathcal{L}}_{v}^{q}=\left(\begin{array}{ccc}
0 & -M_{12} & 0 \\
M_{21} & 0 & 0 \\
0 & 0 & 0
\end{array}\right) & \overline{\mathcal{L}}_{v}^{v}=-\left(\begin{array}{ccc}
N_{11} & 0 & 0 \\
0 & N_{22} & 0 \\
0 & 0 & N_{33}
\end{array}\right) \\
\overline{\mathcal{L}}_{b}^{q}=\left(\begin{array}{ccc}
0 & 0 & 0 \\
0 & 0 & 0 \\
-\lambda \mathbf{B}^{2} & \lambda \mathbf{B}^{1} & 0
\end{array}\right) & \overline{\mathcal{L}}_{b}^{v}=\left(\begin{array}{lll}
0 & 0 & 0 \\
0 & 0 & 0 \\
0 & 0 & 0
\end{array}\right) .
\end{aligned}
$$

In (Earth-fixed) coordinates

$$
\delta \eta_{q}:=\left(\begin{array}{c}
0 \\
\delta \eta_{q}^{1} \\
\delta \eta_{q}^{2} \\
\delta \eta_{q}^{3}
\end{array}\right), \delta \eta_{v}:=\left(\begin{array}{c}
\delta \eta_{v}^{1} \\
\delta \eta_{v}^{2} \\
\delta \eta_{v}^{3}
\end{array}\right) \text { and } \mathbf{A}_{g r}=\left(\begin{array}{c}
0 \\
0 \\
\mathbf{a}_{\mathrm{gr}}
\end{array}\right) \text {. }
$$

The matrices were chosen so that the error system decomposes in four decoupled subsystems:

- the longitudinal subsystem

$$
\left(\begin{array}{l}
\delta \frac{d}{d t} \eta_{q}^{2} \\
\delta \frac{d}{d t} \eta_{v}^{1}
\end{array}\right)=\left(\begin{array}{cc}
0 & M_{21} \\
-2 \mathrm{a}_{\mathrm{gr}} & -N_{11}
\end{array}\right)\left(\begin{array}{l}
\delta \eta_{q}^{2} \\
\delta \eta_{v}^{1}
\end{array}\right)
$$

- the lateral subsystem

$$
\left(\begin{array}{l}
\delta \frac{d}{d t} \eta_{q}^{1} \\
\delta \frac{d}{d t} \eta_{v}^{2}
\end{array}\right)=\left(\begin{array}{cc}
0 & -M_{12} \\
2 \mathbf{a}_{\mathrm{gr}} & -N_{22}
\end{array}\right)\left(\begin{array}{l}
\delta \eta_{q}^{1} \\
\delta \eta_{v}^{2}
\end{array}\right)
$$

- the vertical subsystem

$$
\delta \frac{d}{d t} \eta_{v}^{3}=-N_{33} \delta \eta_{v}^{3}
$$

- the heading subsystem

$\delta \frac{d}{d t} \eta_{q}^{3}=\lambda \mathbf{B}^{3}\left(\mathbf{B}^{1} \delta \eta_{q}^{1}-\mathbf{B}^{2} \delta \eta_{q}^{2}\right)-\lambda\left(\left(\mathbf{B}^{1}\right)^{2}+\left(\mathbf{B}^{2}\right)^{2}\right) \delta \eta_{q}^{3}$.
We can freely assign the eigenvalues of each of the subsystems. We obtained the following non trivial result:

Theorem 6: Consider the dynamics (22). The non-linear observer

$$
\begin{aligned}
\frac{d}{d t} \hat{q}= & \frac{1}{2} \hat{q} * \omega(t)+\left(\overline{\mathcal{L}}_{v}^{q} E_{v}+\overline{\mathcal{L}}_{b}^{q} E_{b}\right) * \hat{q} \\
\frac{d}{d t} \hat{v}= & \hat{v} \times \omega(t)+\hat{q}^{-1} * \mathbf{A}_{g r} * \hat{q}+a(t) \\
& +\hat{q}^{-1} *\left(\overline{\mathcal{L}}_{v}^{v} E_{v}+\overline{\mathcal{L}}_{b}^{v} E_{b}\right) * \hat{q}
\end{aligned}
$$

with

$$
E_{v}=\hat{q} *\left(\hat{v}-y_{v}(t)\right) * \hat{q}^{-1}, \quad E_{b}=\mathbf{B}-\hat{q} * y_{b}(t) * \hat{q}^{-1}
$$

and with the constant gain matrices $\overline{\mathcal{L}}_{v}^{q}, \overline{\mathcal{L}}_{v}^{v}, \overline{\mathcal{L}}_{b}^{q}$ and $\overline{\mathcal{L}}_{b}^{v}$ chosen such that the linear systems (27), (28), (29) and (30), are asymptotically stable, converges locally and exponentially around any system trajectory. The invariant estimation state error obeys an autonomous differential equation (25). The convergence behavior and Lyapunov exponents are completely independent of the system trajectory and of the inputs.

Simulations below indicate that the convergence is far from being only local. We suspect much stronger stability. We conjecture that such non-linear invariant observer is almost globally convergent. It can not be globally convergent because of the following "spin" effect: if $\left(\eta_{q}=1, \eta_{v}=0\right)$ is a locally asymptotically stable steady state for the invariant error equation (25), $\left(\eta_{q}=-1, \eta_{v}=0\right)$ is also a locally asymptotically stable steady-state. From a physical point of view this is not important since $\hat{q}$ and $-\hat{q}$ correspond to the same rotation $R_{\hat{q}}$ in $S O(3)$.

5) Simulations: To obtain realistic values of $\omega, v, a$ and $q^{-1} *$ $\mathbf{B} * q$ all expressed in the body frame, we generated a trajectory of a simplified VTOL-like aircraft. The flight is modeled the following way: initially $q$ is the unit quaternion. Let $k$ denote the downwards vertical axis of the body frame (quaternion $e_{3}$ ) and $P$ the position of the center of mass of the body. We suppose the motion is such that $k$ is always collinear to $\ddot{P}-\mathbf{A}_{g r}$. We suppose $q$ corresponds to the rotation which maps $\mathbf{A}_{g r}$ to $k$ and whose rotation axis is collinear to $\mathbf{A}_{g r} \times k$.

We suppose initially that $P(0)=\dot{P}(0)=\ddot{P}(0)=0 . P(t)$ follows a circular trajectory whose radius is 5 meters, parameterized by the angle $\theta(t)$. The function $t \mapsto P(t)$ is $C^{3}$ with

- For $0 \leq t \leq t_{1}$ we have $0 \leq \theta \leq \pi / 2$ and $\ddot{\theta}(t)=$ $c\left(1-\cos \left(2 \pi t / t_{1}\right)\right)$ where $c=\left(1 / t_{1}^{2}\right)\left(2 \pi^{3} / 2 \pi^{2}+1\right)$ is chosen such that $t_{1}=2 s$;

- For $t_{1} \leq t \leq t_{2}$ we have $\pi / 2 \leq \theta \leq 3 \pi / 2$ and $\ddot{\theta}(t)=0$ with $t_{2}=4.15 \mathrm{~s}$

- For $t_{2} \leq t \leq t_{3}$ we have $3 \pi / 2 \leq \theta \leq 2 \pi$ and $\ddot{\theta}(t)=$ $-c\left(1-\cos \left(2 \pi\left(t-t_{2}\right) / t_{1}\right)\right.$ with $t_{3}=6.15 \mathrm{~s}$.

The drone eventually stops after having followed a circle. The maximum horizontal acceleration is approximately $10 \mathrm{~ms}^{-2}$. Such inverse kinematic model provides realistic values for $a(t)$, $\omega(t), v(t)$ and $q^{-1}(t) * \mathbf{B} * q(t)$ corresponding to this trajectory. We take $\mathbf{B}=[(1 / \sqrt{2}), 0,(1 \sqrt{2})]^{T}$. 

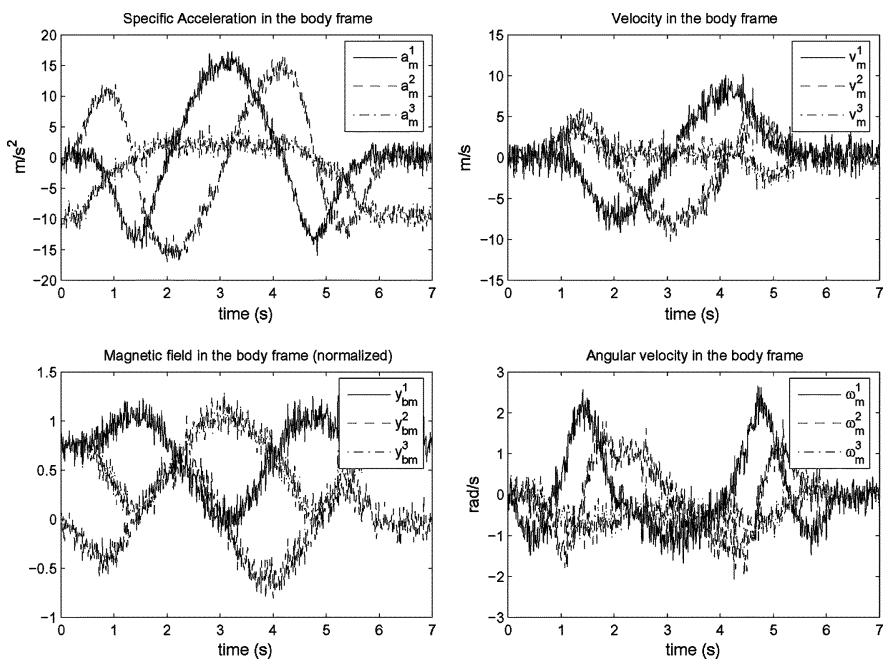

Fig. 1. Measured signals (with noise and bias): specific acceleration $a$, velocity $y_{v}=v$, normalized magnetic field $y_{b}$, and angular velocity $\omega$ in the body frame.
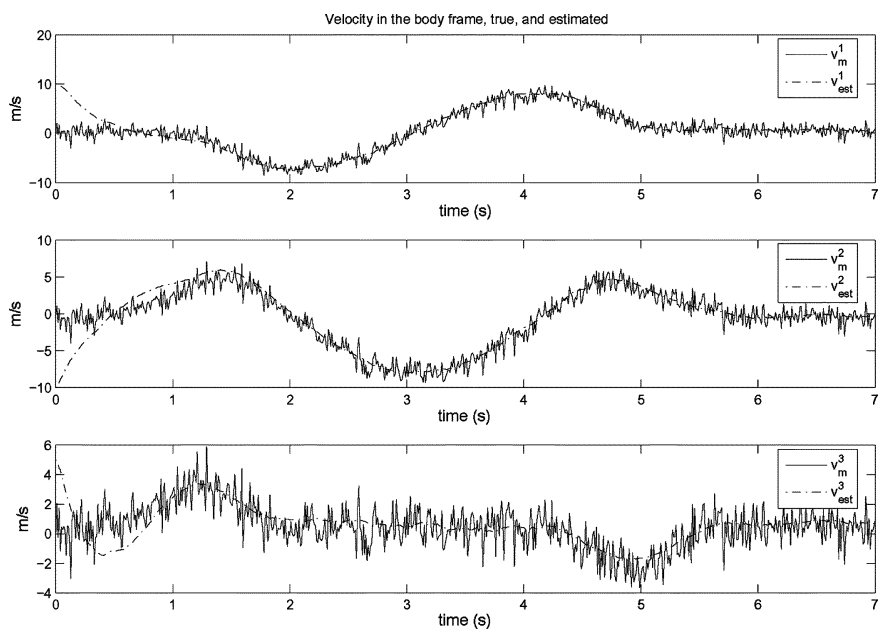

Fig. 2. Measured velocity $v$ (solid line) and estimated velocity $\hat{v}$ (dashed-line) via the invariant observer (31) (with noise and bias).

For the simulations illustrated by Figs. 1-3, the initial conditions are

$\begin{array}{ccc} & \text { True system } & \text { Observer }(31) \\ q_{0} & 1 & \cos (\pi / 3) \\ q_{1} & 0 & \sin (\pi / 3) / \sqrt{(3)} \\ q_{2} & 0 & -\sin (\pi / 3) / \sqrt{(3)} \\ q_{3} & 0 & \sin (\pi / 3) / \sqrt{(3)} \\ v_{1} & 0 & 10 \\ v_{2} & 0 & -10 \\ v_{3} & 0 & 5\end{array}$

That means the initial rotation differs from the true one up to a $2 \pi / 3$ angle. The gains are the following: $M_{12}=M_{21}=0.4$, $N_{11}=N_{22}=4, N_{33}=2$ and $\lambda=4$. With $\mathbf{a}_{g r}=10 \mathrm{~ms}^{-2}$, the poles of the longitudinal and the lateral subsystems are: $-2(1 \pm$ i) $s^{-1}$ and the poles of the vertical and heading subsystems are: $-2 s^{-1}$.
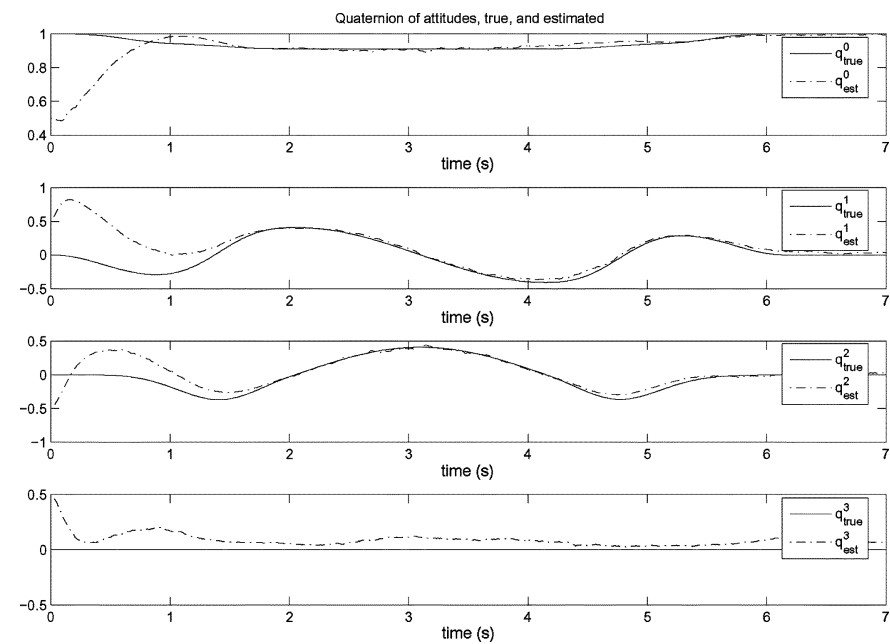

Fig. 3. Orientation $q$ (solid line) and estimated orientation $\hat{q}$ (dashed-line) via the invariant observer (31) (with noise and bias).

The measured signals are noisy and biased: some high frequencies and some bias are added to the signals $\mathbf{a}(t), \omega(t)$, $v(t)$ and $q^{-1} * \mathbf{B} * q(t)$ in order to represent the imperfections of the sensors. The noisy and biased signals are defined by: $\mathbf{a}_{m}(t)=\mathbf{a}(t)+0.5[1,-1,1]+\sigma_{1}$, and $\omega_{m}(t)=\omega(t)+$ $4 \pi / 360[1,-1,1]+0.25 \sigma_{2}$, and $v_{m}(t)=v(t)+0.5[1,-1,1]+$ $\sigma_{3}$, and $y_{b m}(t)=y_{b}(t)+0.05[1,-1,1]+0.1 \sigma_{4}$, where the $\sigma_{i}$ are independent normally distributed random 3 -D vectors with mean 0 and variance 1 , and $\mathbf{a}(t), \omega(t), v(t), q^{-1} * \mathbf{B} * q(t)$ are the perfect and smooth signals calculated from the VTOL-type drone dynamics. These simulations show that the asymptotic observer (31) admits a large attraction region and is quite robust to measurement noise and bias.

\section{CONCLUSION}

A theory of symmetry-preserving observers has been developed. It is mainly composed of: a constructive method to find all the symmetry-preserving pre-observers (see Section II-D), and a constructive method to find an invariant error between the actual state of the system and its estimate (see Section II-F). The resulting invariant error equation simplifies the convergence analysis. Although we have only provided examples to support these claims, we believe the following properties of a symmetry-preserving observer can be expected.

- The observer naturally inherits important geometric features of the system (e.g., the observed concentrations in Example III-B are positive, the observed quaternion in Example III-C has unit norm).

- Constant gains can be chosen thanks to the usual linear techniques (see Section II-E) to achieve local convergence. In the case where the group and the state space can be identified, one can possibly expect local convergence around every trajectory of the system, and not only around its equilibrium points or "slowly-varying" trajectories [5].

- As the observer respects the geometry of the system, the global behavior tends to be better and the region of attraction larger (compared e.g. to a Luenberger observer).

Moreover, we believe the invariance property of such an observer is often desirable from an engineering point of view, if 
not from an aesthetic one. Finally the method presented in this paper can at least be seen as a useful new tool in the not-so-full toolbox of design methods for non-linear observers, since many physical and engineering systems possess symmetries.

\section{REFERENCES}

[1] N. Aghannan, "Contrôle de Réacteurs de Polymérisation, observateur et invariance," Ph.D. dissertation, Ecole des Mines de Paris, Paris, France, Nov. 2003.

[2] N. Aghannan and P. Rouchon, "On invariant asymptotic observers," in Proc. 41st IEEE Conf. Decision Control, 2002, vol. 2, pp. 1479-1484.

[3] N. Aghannan and P. Rouchon, "An intrinsic observer for a class of lagrangian systems," IEEE Automat. Control, vol. 48, no. 6, pp. 936-945, Jun. 2003.

[4] R. Aris and N. R. Amundson, "An analysis of chemical reactor stability and control-i,ii,iii," Chem. Eng. Sci., vol. 7, pp. 121-155, 1958.

[5] S. Bonnabel, P. Martin, and P. Rouchon, Non-Linear Symmetry-Preserving Observers on Lie Groups [Online]. Available: http://arxiv.org/ abs/0707.2286 2007

[6] S. Bonnabel, P. Martin, and P. Rouchon, "Groupe de lie et observateur non-linéaire," in Proc. Conf. Int. Francophone d'Automatique (CIFA'06), Bordeaux, France, Jun. 2006, [CD ROM].

[7] S. Bonnabel, P. Martin, and P. Rouchon, "A non-linear symmetry-preserving observer for velocity-aided inertial navigation," in Proc. Amer. Control Conf. (ACC06), Jun. 2006, pp. 2910-2914.

[8] S. Bonnabel and P. Rouchon, "On invariant observers," in Control and Observer Design for Non-Linear Finite and Infinite Dimensional Systems. New York: Springer, 2005, vol. 322, pp. 53-66.

[9] G. Creamer, "Spacecraft attitude determination using gyros and quaternion measurements," J. Astronaut. Sci., vol. 44, no. 3, pp. 357-371, 1996.

[10] F. Fagnani and J. Willems, "Representations of symmetric linear dynamical systems," SIAM J. Control Optim., vol. 31, pp. 1267-1293, 1993.

[11] J. W. Grizzle and S. I. Marcus, "The structure of non-linear systems possessing symmetries," IEEE Trans. Automat. Control, vol. AC-30, no. 3, pp. 248-258, Mar. 1985.

[12] T. Hamel and R. Mahony, "Attitude estimation on so(3) based on direct inertial measurements," in Proc. Int. Conf. Robot. Automat. (ICRA'06), 2006, pp. 2170-2175.

[13] R. Mahony, T. Hamel, and J.-M. Pflimlin, "Complimentary filter design on the special orthogonal group $\mathrm{SO}(3)$, , in Proc. IEEE Conf. Decision Control (CDC'05), Seville, Spain, Dec. 2005, pp. 1477-1484.

[14] D. H. S. Maithripala, W. P. Dayawansa, and J. M. Berg, "Intrinsec observer-based stabilization for simple mechanical systems on lie groups," SIAM J. Control Optim., vol. 44, pp. 1691-1711, 2005.

[15] P. Martin, P. Rouchon, and J. Rudolph, "Invariant tracking," ESAIM: Control, Optim. Calculus Variations, vol. 10, pp. 1-13, 2004.

[16] P. J. Olver, Equivalence, Invariants and Symmetry. Cambridge, U.K.: Cambridge Univ. Press, 1995.

[17] P. J. Olver, Classical Invariant Theory. Cambridge, U.K.: Cambridge Univ. Press, 1999.

[18] J. Ortega and R. S. Ratiu, Momentum Maps and Hamiltonian Reduction. Basel, Switzerland: Birkhauser, 2000.
[19] W. Respondek and I. A. Tall, "non-linearizable single-input control systems do not admit stationary symmetries," Syst. Control Lett., vol. 46, pp. 1-16, 2002.

[20] A. J. van der Schaft, "Symmetries in optimal control," SIAM J. Control Optim., vol. 25, pp. 245-259, 1987.

[21] M. W. Spong and F. Bullo, "Controlled symmetries and passive walking," IEEE Trans. Automat. Control, vol. 50, no. 7, pp. 1025-1031, Jul. 2005.

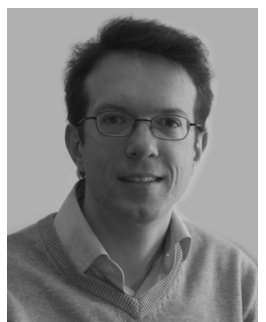

Silvère Bonnabel was born in Marseille, France, in 1981. He received the M.S. and Ph.D. degrees in mathematics from Ecole Nationale Supérieure des Mines de Paris (ENSMP), Paris, France, in 2004 and 2007, respectively.

He was a Postdoctoral Fellow at the University of Liège, Liège, Belgium, in 2008. He has worked on an industrial application (chemical reactor). His research interests include geometrical methods for estimation problems.

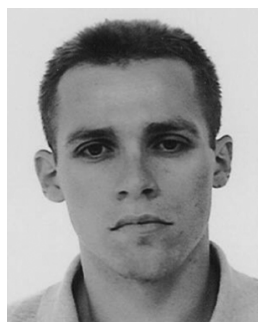

Philippe Martin received the Ph.D. degree in mathematics and control from the Ecole des Mines de Paris, Paris, France, in 1992.

He is currently a Senior Researcher with the Ecole des Mines de Paris. From 1993 to 1994, he visited the Center for Control Engineering and Computation, University of California at Santa Barbara and the Department of Mathematics, University of North Carolina at Chapel Hill. Since 2000, he has also been holding a part-time position of Associate Professor at Ecole Centrale Paris. His interests include theoretical aspects of nonlinear control and their applications to industrial control problems.

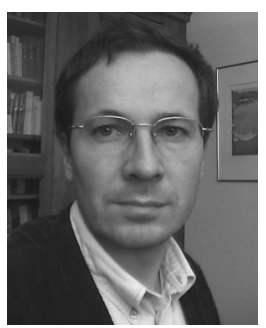

Pierre Rouchon was born in Saint-Etienne, France, in 1960. He received the M.S. degree from Ecole Polytechnique, Palaiseau, France, in 1983 and the $\mathrm{Ph} . \mathrm{D}$. degree in chemical engineering from Ecole des Mines de Paris, Paris, France, in 1990, and the habilitation à diriger des recherches degree in mathematics at the University Paris-Sud Orsay, in 2000.

From 1993 to 2005, he was an Associate Professor in applied mathematics with École Polytechnique. From 1998 to 2002, he was the head of the Centre Automatique et Systémes of École des Mines de Paris. He is now a Professor at Ecole des Mines de Paris. His fields of interest include the theory and applications of dynamical systems, nonlinear control, and in particular differential flatness and its extension to infinite dimensional systems. He has worked on many industrial applications such as distillation columns, electrical drives, car equipments, chemical reactors. One of his recent field of interest is relative to the control and estimation of closed and open quantum systems. 